\title{
Accurate Distance Estimation Using Fuzzy based combined RSSI/LQI Values in an Indoor Scenario: Experimental Verification
}

\author{
Talmai Oliveira, Madhanmohan Raju, and Dharma P. Agrawal \\ School of Computing Sciences and Informatics, \\ Center for Distributed and Mobile Computing, \\ University of Cincinnati, Cincinnati, OH, 45221-0008 (USA) \\ E-mail: oliveitb@mail.uc.edu,rajumn@mail.uc.edu,dpa@cs.uc.edu
}

Received: July 30, 2012 Accepted: November 10, 2012 Published: December 16, 2012

DOI: $10.5296 / n p a . v 4 i 4.2173$

URL: http://dx.doi.org/10.5296/ npa.v4i4.2173

\begin{abstract}
The received signal strength indicator (RSSI) and the link quality indicator (LQI) are the metrics that are commonly available in commercial off-the-shelf (COTS) sensor hardware. The former has been widely regarded as the main source for distance estimation and node localization. However, experimentally RSSI has been shown to behave in an inconsistent manner, even in ideal scenarios, and serve at best as bounds for distances. The latter is effectively a measure of chip error rate, and can be used to identify higher quality transmissions, and the combination RSSI/LQI can be expected to make more precise estimates with the tradeoff of increased delay and estimation cost. In this paper, we describe our distance estimation system that uses these two metrics and test our hypothesis purely through experimental measurements using sensor nodes. Results indicate that such a combination of metrics can be used to provide a tighter bound on the range of estimated distances. We then quantify the improvement in distance estimation by relying on these two metrics. Through a unique classification using fuzzy logic and TBM, we developed an algorithm that is capable of precise distance estimation within the range of $100 \mathrm{~cm}$ to $400 \mathrm{~cm}$, on at least $80 \%$ of the times while reaching accuracy as high as $100 \%$.
\end{abstract}

Keywords: Indoor Distance Estimation, Link Quality Indicator (LQI), Received Signal Strength Indicator (RSSI), Transferable Belief Model (TBM), Wireless Sensor Networks (WSN). 


\section{Introduction}

The received signal strength indicator (RSSI) has largely been perceived by the wireless sensor network (WSN) community as an inadequate estimator and metric for determining link communication quality between any two neighboring nodes. In indoor radio communication, this problem is exacerbated by the known fact that the transmitted signal suffers from multipath fading due to reflection, refraction and scattering of radio waves by structures inside a building [1]. Performance of communication is therefore seriously degraded in indoor scenarios. Unfortunately, not much can be done to eliminate the problem, even when multipath medium is well characterized and nodes adequately model the channel to reduce the effect of these disturbances. Later experimental results showed that while detecting good links is possible with RSSI, link quality estimates with imperfect communication channels are not accurate [2].

But, besides being used for link quality assessment, RSSI has been suggested by the wireless network community to be used as a distance estimator and node localization mechanism [3-6]. Even though RSSI was not meant for this purpose, the idea of providing this information with no added cost to higher communication layers is very attractive. RSSI is a measure of $\mathrm{dBm}$, which is 10 times the logarithm of the ratio of the received power $(\mathrm{P})$ and the reference power (PREF). Power at the receiver is inversely proportional to the square of the distance. Therefore, this is a relatively straightforward conversion, and RSSI can be used as an indicator of distance. But, since RSSI is sensitive to the channel noise, interference, reflections and attenuations, it suffers from the antenna variability. Additional experiments have confirmed that RSSI can't be completely relied on as an absolute metric [7].

For this reason, distance estimation and localization has been observed to be a very challenging problem [8,9]. Since RSSI patterns are very complex, even the slightest movement significantly vary the received signal [10]. For example, for each given RSSI level there could be many, even distant locations in the room where the same value is received. This means that RSSI and the sender-announced power level does not necessarily reflect an unique theoretical value. Additional environmental factors such as temperature and humidity have been shown to interfere with RSSI readings as well [11]. So due to a strong non-linear characteristic of RSSI, any localization or distance estimation method that relies on previously measured RSSI fingerprint levels, may fail even for approaches that apply filters or signal processing [12-14]. Comprehensive studies of indoor RSSI have been conducted in $[10,15]$ where the authors show that the only way to improve the accuracy would be through a more complex model of the RSSI behavior. What has been generally accepted is that, while experimentally RSSI has been shown to behave in an inconsistent manner (even in ideal an scenario [16]), at best, it could be used as bounds for the distance [11].

All of the previous research results are not surprising, and are even somewhat intuitively anticipated. Basing such an important decision on a single value leads to limited accuracy and distorted predictions about future values. What practitioners have then tried to explore is the combination of RSSI with additional metrics. The link quality indicator (LQI), which is effectively a measure of chip error rate commonly available in COTS sensor hardware, has 
been shown to be highly correlated with both RSSI [17] as well as with packet reception rate (except when operating at the edge of receiver sensitivity). Such a complementary combination shows that while RSSI can be an attractive agile link estimator, LQI can make more precise estimates with increased delay and estimation cost.

Additional precision and positioning accuracy can be achieved by using other features besides LQI, such as both the short and long-term characteristics of the link quality, as well as information about dynamic variation and trends of the signal [17, 18]. For example, in [18], four distinct quality metrics are used. The tradeoff is complexity, since by adding metrics there is an increase in the calculation time. Some research has even been done to replace LQI altogether [19]. This, however, would require additional effort and complexity as well, as for this reason we do not consider it. The devices might also require the physical presence of additional components and interfaces.

\subsection{Related Work}

A distributed algorithm has been proposed [9] that first identifies neighboring devices with the highest RSSI and LQI readings. A coordinator is elected which is responsible for calculating an average of the neighboring device's values. With this information, nodes then individually use a weighted centroid localization algorithm which results in a good accuracy. However, high cost of running a distributed leader election protocol and collection and generation of statistics of the RSSI and LQI are ignored. While the idea of reaching a consensus is good, we believe that this has to be done only on a local level, relying only on the nearest neighbors.

A maximum likelihood estimation (MLE) algorithm [20] is proposed that deals with the noise better than a direct triangulation algorithm. The authors of [21] go one step further and propose both a hardware implemented MLE as well as a software implemented triangulation localization algorithm. They performed experimental test for both the indoor and the outdoor applications. However, these research papers require reference devices with previously known coordinates and it requires a substantially long and complex calibration phase.

While [22] does not introduce any distance estimation algorithm, it proposes an RSSI approximation method that uses Newton interpolation polynomial (NIP) to continuously approximate RSSI with limited sample points. According to the authors, NIP is smooth in the domain and avoids continuity problems that arise with other interpolation methods. In our opinion, this is too complex and cumbersome to calculate on a sensor, and depending on previously deployed reference devices is not ideal.

The "too complex" argument is also applicable to the work of [12] which uses Sigma-Point Kalman Smoothers; to the work of [4] which uses a centralized server to solve individual devices' log-distance path loss equations and runs a genetic algorithm. The work of [18] proposes a combination of metrics for distance estimation and localization and uses four distinct metrics to represent a better model of the short and long-term quality of the link as well as information on the dynamic variation and the trend of the communication signal.

While fuzzy logic based distance estimation has been done before [23, 24], indoor usage 
and results are still lacking. In this paper, we propose the combination of fuzzy logic and the transferable belief model, a completely novel idea and, to our knowledge, no other similar work has been done.

\subsection{Main Contributions}

In this work, we present an ongoing study of indoor distance estimation. First, we describe our distance estimation system that uses only the RSSI and LQI values. We test whether our hypothesis is correct by creating a practical experimental setup and by performing real-life measurements by deploying real sensors. A simplified fingerprinting system is developed by evaluating the experimental analysis of RSSI and LQI readings over different power levels with limited interference. We do not consider any specific power level of the transmitting device, which means that when analyzing the values, receiving device initially infers a very large set of possible distances. While our subsequent steps rely on our measured experimental data in order to estimate the distance, we remind the reader that what we are proposing is a comprehensive solution that can be easily adapted to different types of sensor hardware and RSSI/LQI levels. Therefore, independent of the environment, the idea remains valid.

With the fingerprint of the measured data determined, we use the RSSI as input to determine all possible distances from the measured data. We then further reduce this set of distances by considering the LQI value received. Our experimental results seem to provide tangible proof that this simple idea can significantly increase correctness of the distance estimation, reducing the possible number of distances. However, as previously mentioned, any practical system cannot just rely on fixed thresholds, nor on filters or signal processing for estimation. Therefore, after the transformation, we execute a distributed consensus protocol between the communicating nodes, selecting the distance with the maximum likelihood. Devices exchange their estimated distance sets and execute a distributed protocol. The process of selecting the distance with a maximum likelihood of occurrence goes through a set of Fuzzy Logic [25] rules before being combined with the help of the Transferable Belief Model (TBM) [26]. This allows us to model and combine evidence in order to make a better decision. It generalizes the Bayesian approach without the need of previously known probabilities. Therefore, it is able to deal with redundant and conflicting information since it is based on the idea of building beliefs on a subjective knowledge.

Experimental results of our proposed novel approach is shown to correctly infer distances between two devices in the range of $100 \mathrm{~cm}$ to $400 \mathrm{~cm}$ in indoor scenario, at least for $80 \%$ of the times, reaching an accuracy as high as $100 \%$.

\section{Analyzing RSSI and LQI as distance estimators}

In this Section, we describe our distance estimation system that uses the received signal strength indicator (RSSI) and the link quality indicator (LQI) metrics. We test our hypothesis purely through experimental measurements using sensor nodes. Results indicate that when the LQI is high, our algorithm is capable of significantly enhancing the accuracy of correctly estimating the distance between two communicating motes. 


\section{$\Lambda$ Macrothink}

\subsection{Experimental Setup}

We conducted experiments both inside and outside of our lab to determine how RSSI and LQI vary with different distances and power levels. Emphasis was placed on determining how reliable these two attributes could be for localization algorithms. The RSSI is the measured power (in $\mathrm{dBm}$ ) of the received radio frequency (RF) signal. The LQI is a link quality metric defined in the 802.15.4 standard, and can be viewed as the chip error rate. During our experiments, emphasis was placed on determining how reliable these two attributes could be for the distance estimation algorithm.

The sensor hardware used is Crossbow's TelosB mote (TPR2420CA) that has an integrated IEEE 802.15.4/ZigBee compliant RF transceiver (CC2420 chipset). Inverted-F antenna was employed whose gain is measured to be $+3.3 \mathrm{~dB}$. The CC2420 operates in the $2.4 \mathrm{GHz}$ ISM band with an effective data rate of 256kbps. It has 16 channels with each one taking over $3 \mathrm{MHz}$ bandwith with a center of frequency separation of $5 \mathrm{MHz}$ between the adjacent channels. The encoding scheme used in the CC2420 encodes 32 chips for a symbol of 4 bits. It also uses an advanced orthogonal quadrature phase shift keying (OQPSK) and direct sequence spread spectrum (DSSS) to modulate the data [9, 27].

The CC2420 stores both the RSSI and the LQI for every received packet. This is measured over the first 8 symbols (32 bits) of a received packet. It is important to state that the standard only requires that the reported RSSI values should be linear and within $\pm 6 \mathrm{~dB}$ of the actual RSSI values. However, this is a wide error margin [28]. The CC2420 only calculates the LQI on the received packets, but continuously measures and calculates the RSSI. This allows a device to calculate the noise floor when there are no transmissions. LQI ranges from 50 to 110 and respectively corresponds to the minimum and maximum quality frames. The LQI measurement represents a correlation between the received symbol and the decoded symbol after appropriate radio decoding has been applied.

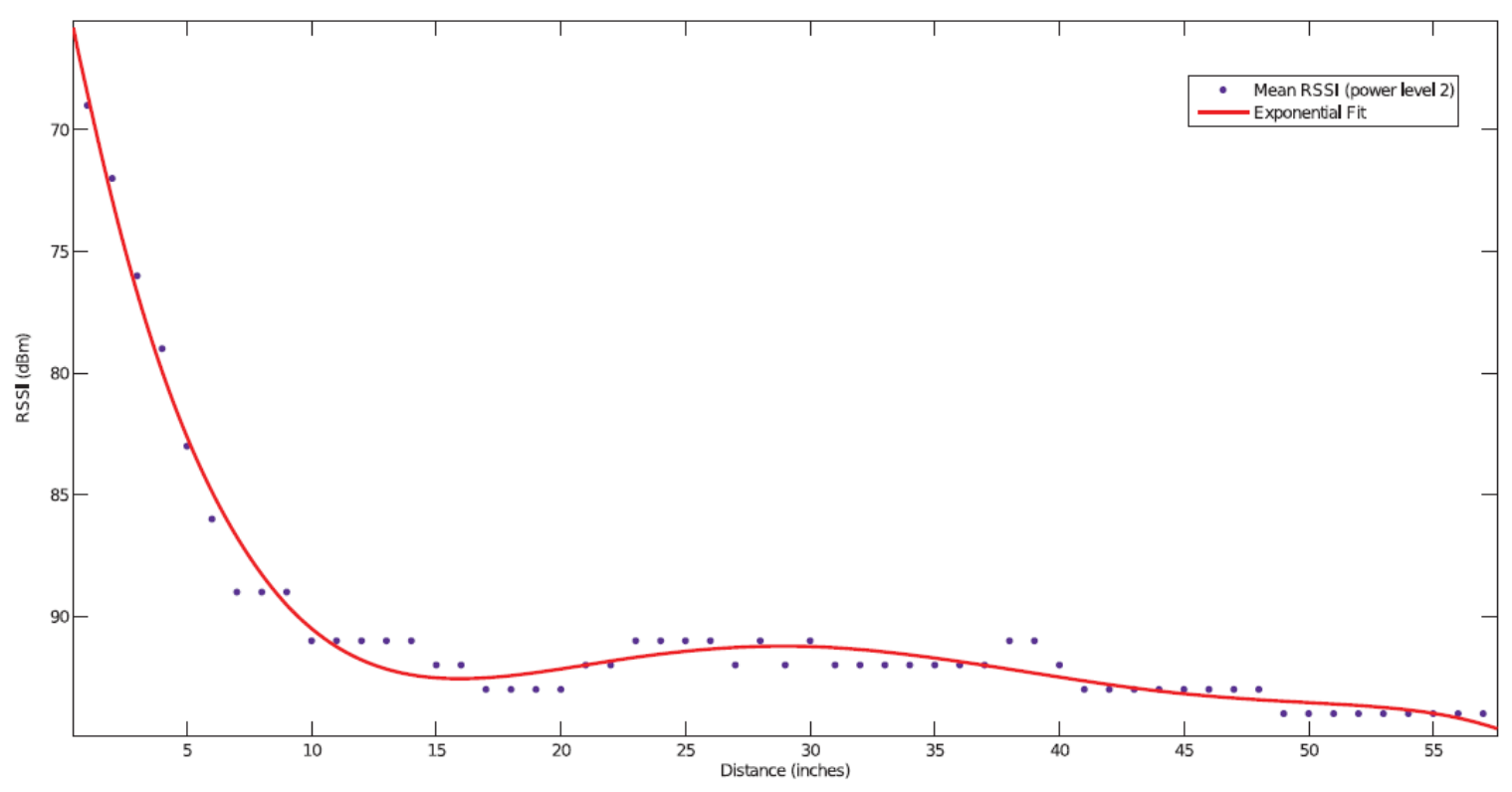

Figure 1. Outdoor Mean RSSI Measurements and Curve Fit. 
Much like previous research with RSSI [16], we placed emphasis on creating an ideal environment in which we could conduct the experiments. At each of the distance, RSSI and LQI are measured for approximately 30 seconds at every 250 milliseconds. On the receiver side of the experiments, the mote were connected via USB to a laptop which was raised to a height of 6 inches from the ground. The sender mote was also raised to the same level from the ground. Both the sender and receiver maintained line of sight transmissions throughout the experiments. The path between the sender and the receiver was clear from any sort of hindrance. This significantly reduced interference. However, we can not state that interference was zero. The motes were programmed in TinyOS to send and receive beacon signals between each other. RSSI and LQI values were calculated from the received beacon signals according to the specified equations in the sensor mote's datasheet. Although consistently checked, the AA batteries never needed to be replaced throughout the span of the experiments.

In this Section, we will discuss both indoor as well as outdoor measurements and results. We remind the reader that the second part of our work focuses solely on the open problem of indoor distance estimation. For the indoor environment, for each of the transmission power levels, RSSI and LQI value were recorded while increasing the distance between the sender and the receiver one centimeter at a time, as far as there was a connection between the nodes. In this set of experiments, we were able to vary the power level from 1 to 7 . The same experiment was repeated in outdoors on a soccer field (using inches as the measurement unit) where we varied the distance between the nodes and the power level variation from 1 to 31 . The difference in power levels from indoor to outdoor occurred due to the transmission range. Anything greater than a power level of 7 indoors would already transmit a message over a larger distance than the longest hall in any of the university's buildings.

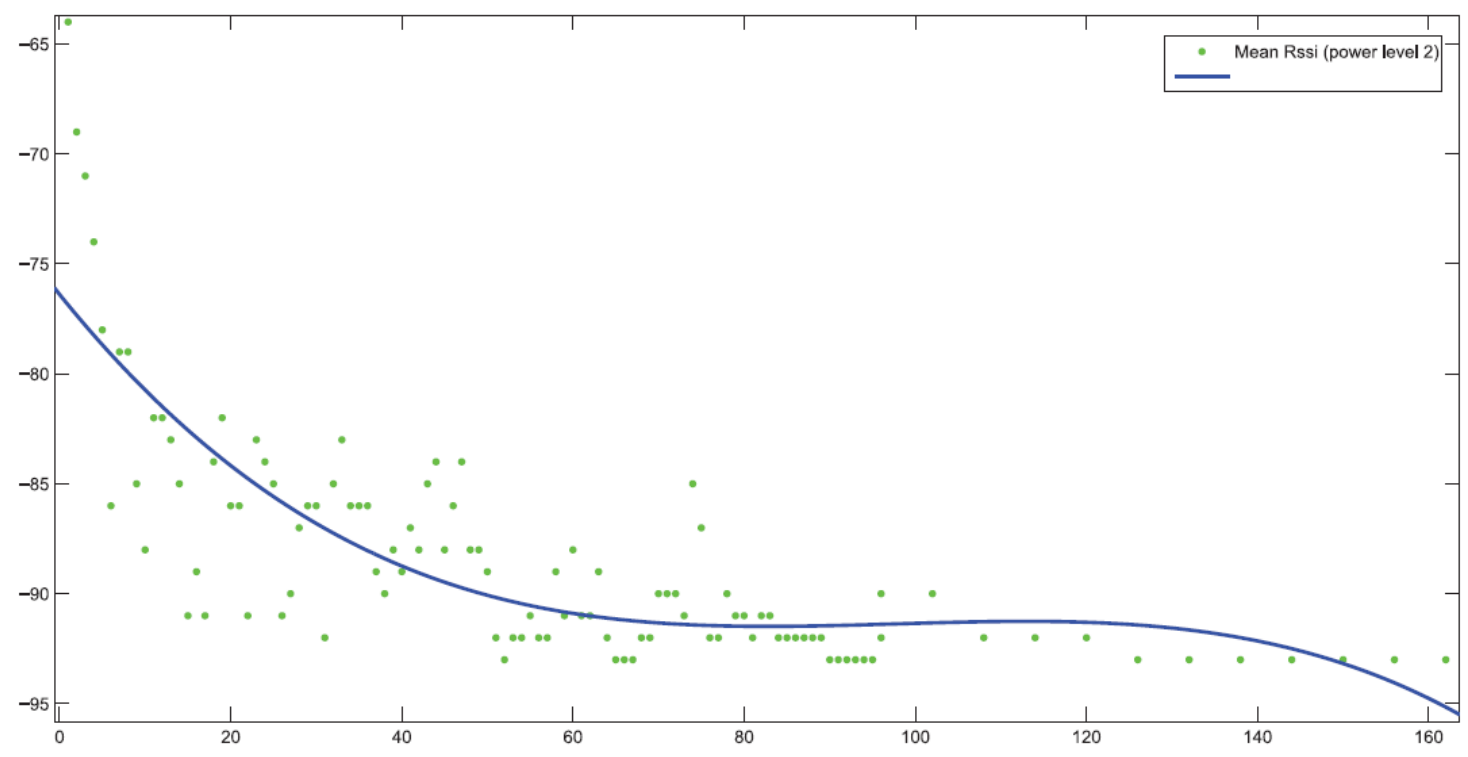

Figure 2. Indoor Mean RSSI Measurements and Curve Fit. 


\section{Macrothink}

\subsection{Analysis of Measurements and Curve Fitting}

For each transmission power level and distance, we calculate the mean of the RSSI at the receiver. We then identify an exponential curve to fit the data. We also ran this step off-line using MATLAB and provided a simple lookup table for any real-time calculations. The curve fits for power level 2 in outdoor experiments can be seen in Fig. 1. The indoor data for the same power level can be seen in Fig. 2.

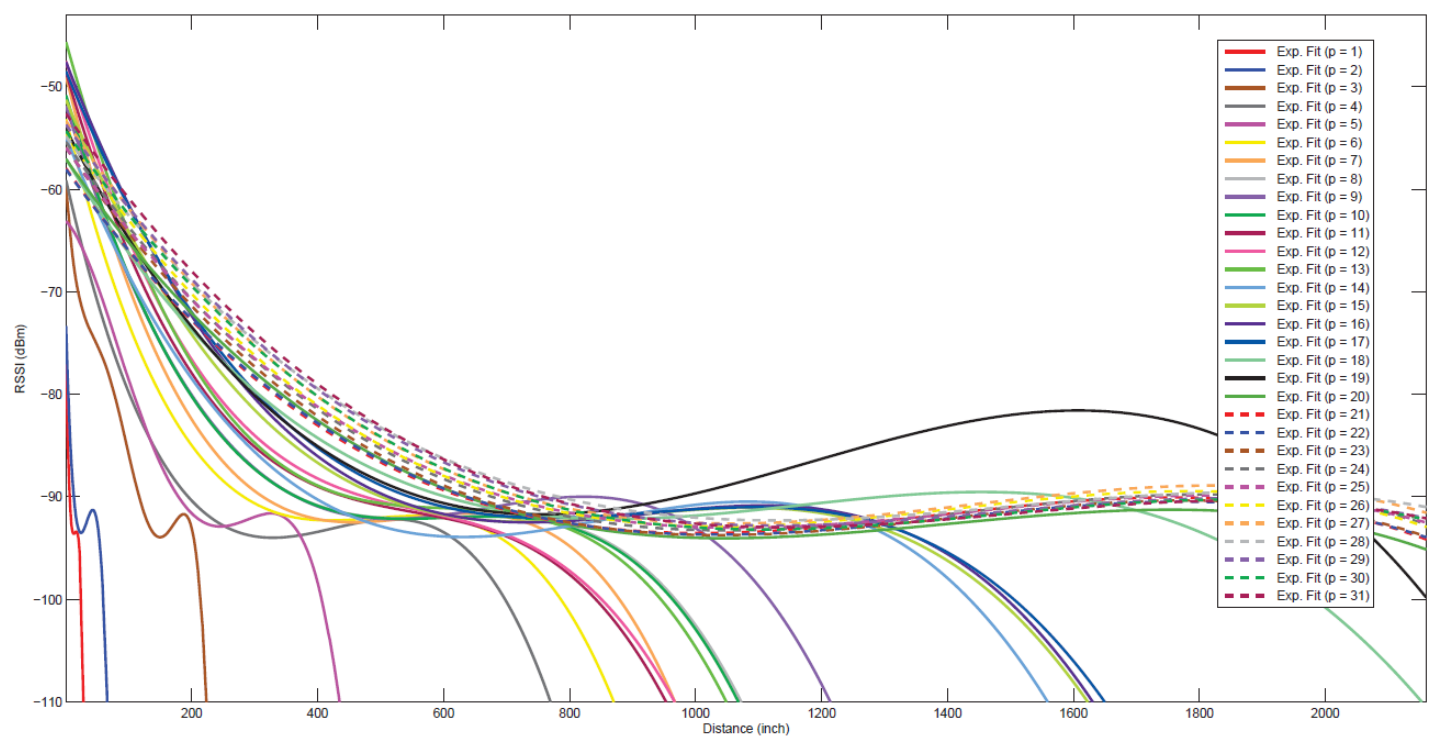

Figure 3. Outdoor RSSI and Curve Fits for Different Power Levels.

Instead of plotting all possible power level, we have considered all possible power levels at once. RSSI values and distances when plotted on the same graph, can be seen in Fig. 3 and Fig, 4. Respectively for outdoor and indoor. Notice that for the same RSSI value, a number of different distances and powers can be assumed.

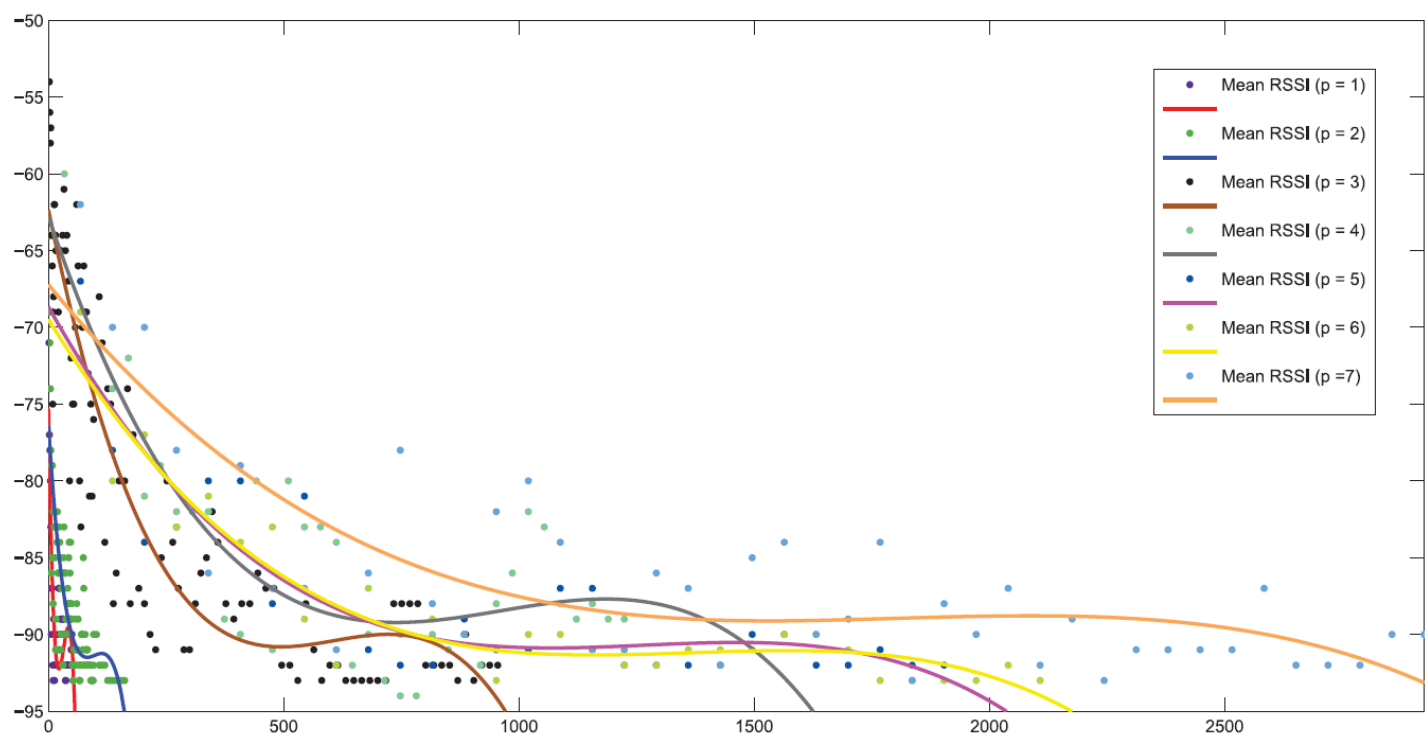

Figure 4. Indoor RSSI and Curve Fits for Different Power Levels. 
As an example, consider that in an indoor transmission, the received node identified RSSI to be $-93 \mathrm{dBm}$. By looking this up on a pre-calculated table, the mote can determine the distance of the transmitting mote to be $[10,13,36,37]$. In fact, if the mote relied only on the RSSI value, considering the number of unique distances that each of the RSSI value would provide, we get a histogram similar to the one seen in Fig. 5. Though the graph shows a few instances (7 to be exact) where only one possible distance/power could be assumed, it also demonstrates many sets with 69 unique distances from which the mote could assume the location of transmitter.

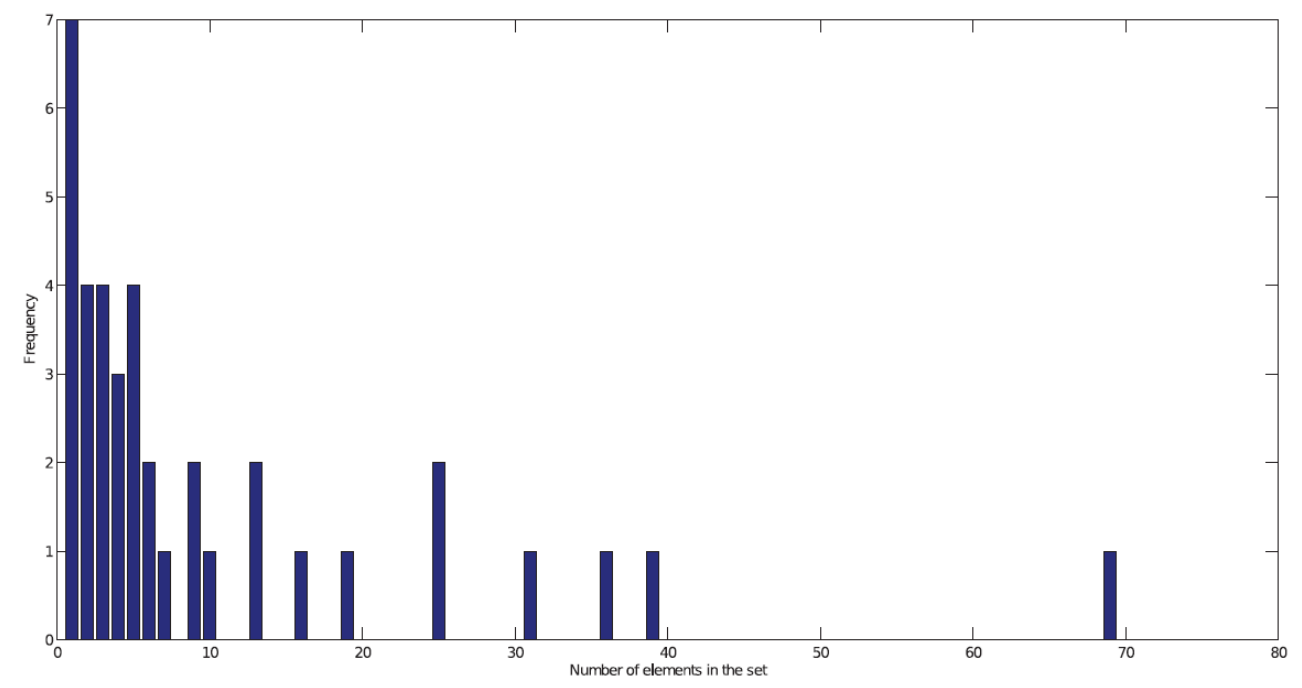

Figure 5. Histogram of the Size of the Possible Distances at Receiver when Experiments where done Indoors.

Fig. 6 shows a similar behavior (this time related to the outdoor measurements). Clearly, some kind of filtering technique needs to be employed in order to reduce the number of possible guesses made by the sensor mote.

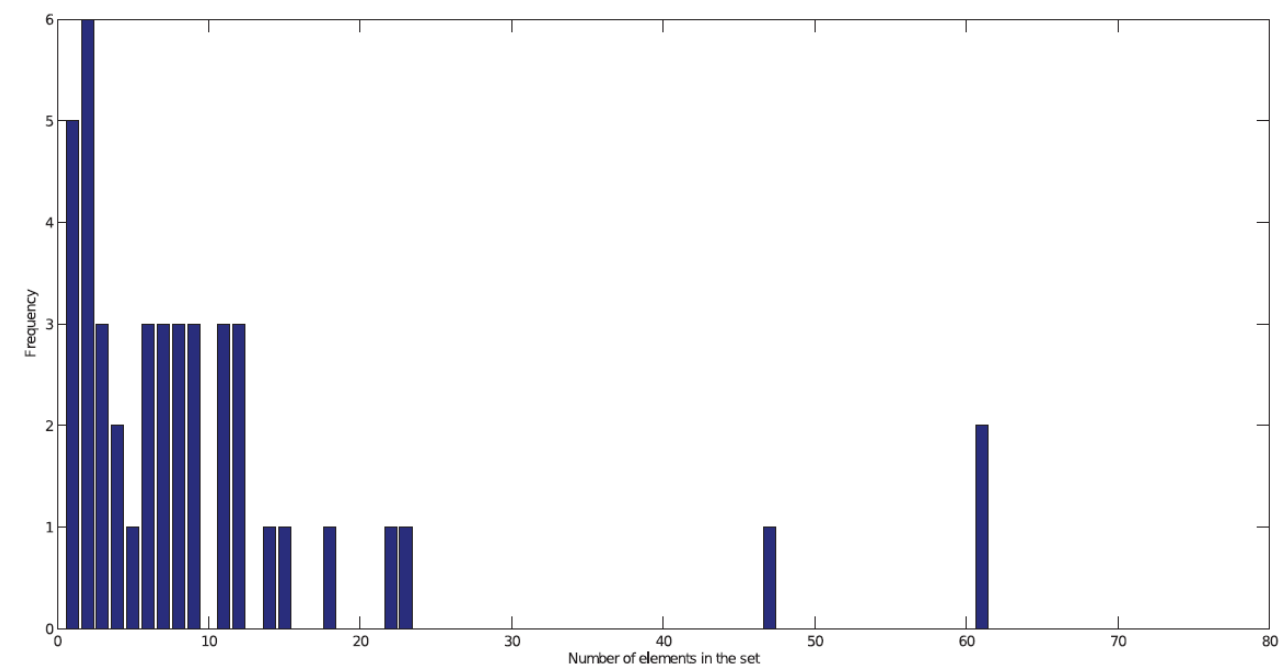

Figure 6. Histogram of the Size of the Possible Distances at Receiver when Experiments where done Outdoors. 


\subsection{Considering LQI for Better Distance Estimation}

The idea behind using both the RSSI and the LQI for a better distance estimation came from our analysis of the experimental data we collected. We discovered that, in an ideal scenario, the LQI could be used to filter out possible distances, since for certain combinations of power vs. distances, the expected best case of LQI could immediately indicate a (possibly) infeasible situation. So, to test our hypothesis, in a similar manner to the RSSI studies, we analyzed the experimental measured LQI data for the same dataset from the previous graphs. We calculated the mean and executed another round of curve fitting algorithms.

An example of the results can be seen in Fig. 7 for power level 3, where an LQI value of 100 (in an ideal environment) only occurs if the distance is less than $300 \mathrm{~cm}$. Therefore, if a possible tuple of estimated distances were $[400 \mathrm{~cm}$, power level 3], then for LQI $=100$, that tuple could be easily discarded

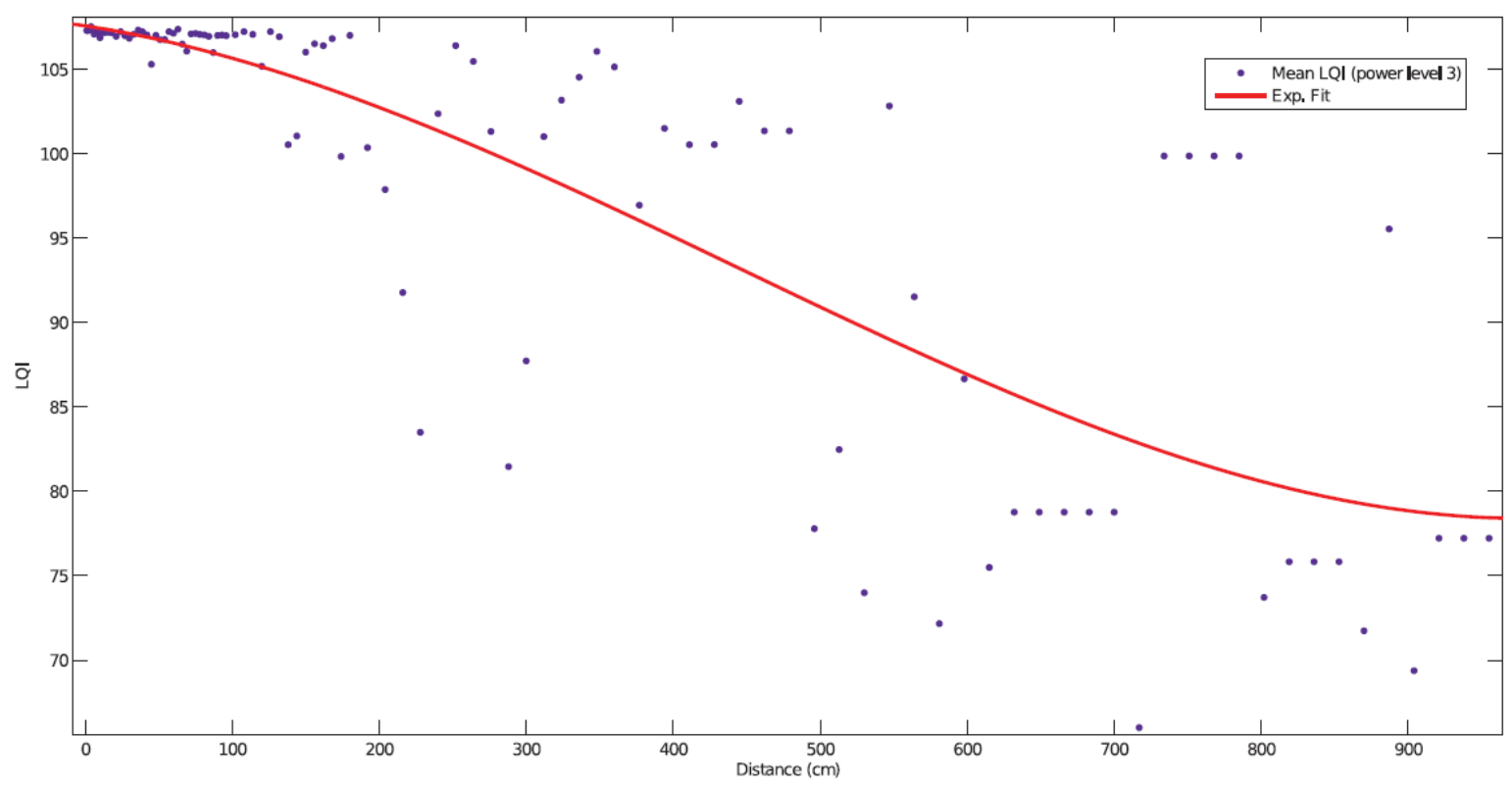

Figure 7. Indoor Mean LQI Measurements and Curve Fit.

Fig. 8 presents all the curve fits for indoor LQI.

Our emphasis here is not on the exact values, but rather how a simple filtering process can be established based on given measurements from RSSI and LQI, such that it increases the precision of distance estimation. For that reason, we will not show the outdoor measurements values, as they will neither add nor delete from our objective. 


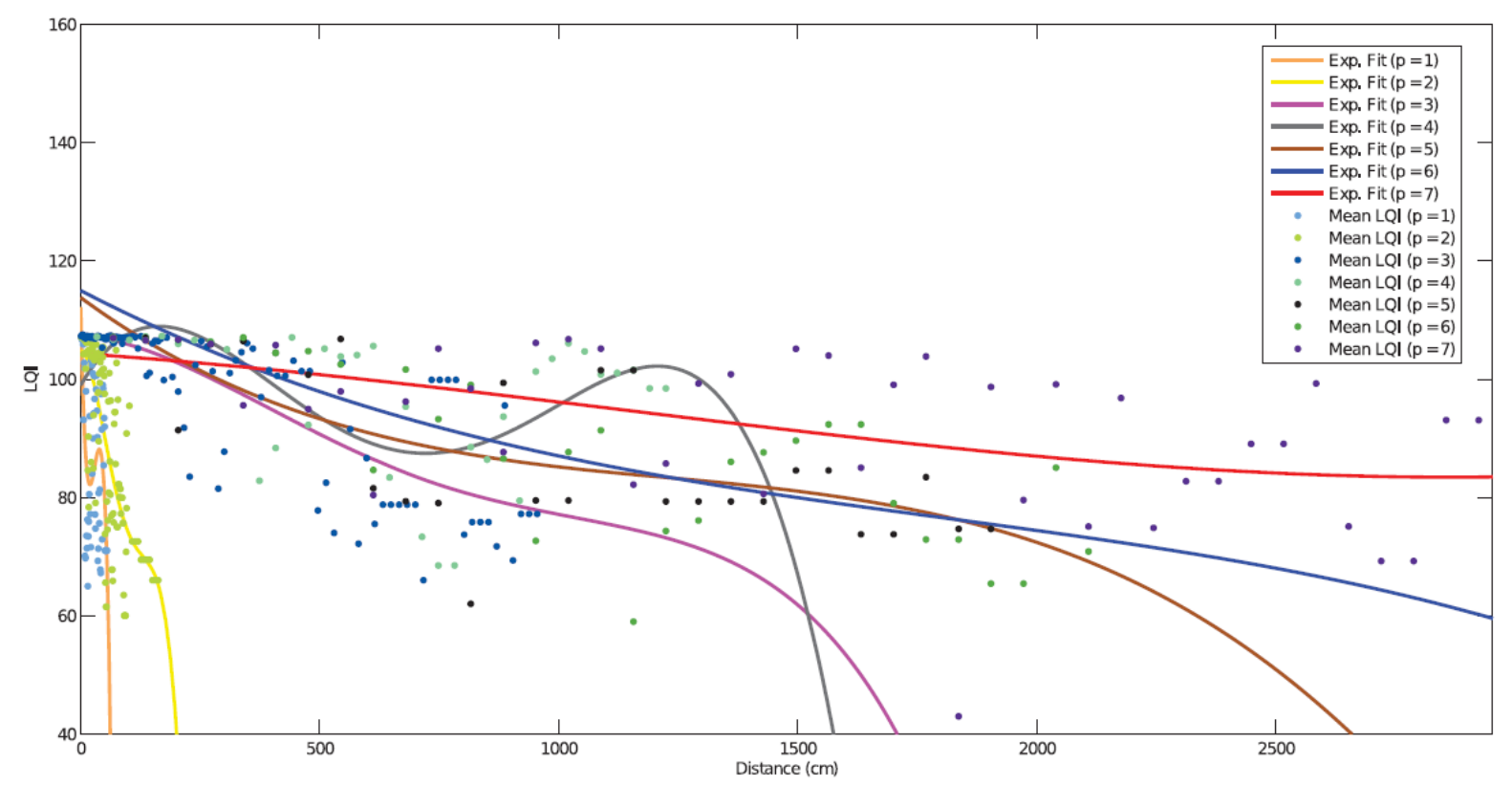

Figure 8. Indoor LQI for Different Power Levels and their Curve Fits.

\subsection{Combining the two Metrics}

So, given the RSSI and LQI values, the sensor mote would first identify the set of all possible distances and the corresponding expected power level. This set would then be further filtered by using the LQI values (fits formed from LQI) and by analyzing the expected results. Simulations of RSSI $=-100 \mathrm{dBm}$ and LQI $=80$ resulted initially in the histogram seen in Fig. 9(a). After applying our simple filtering mechanism, a new histogram can be obtained as shown in Fig. 9(b).

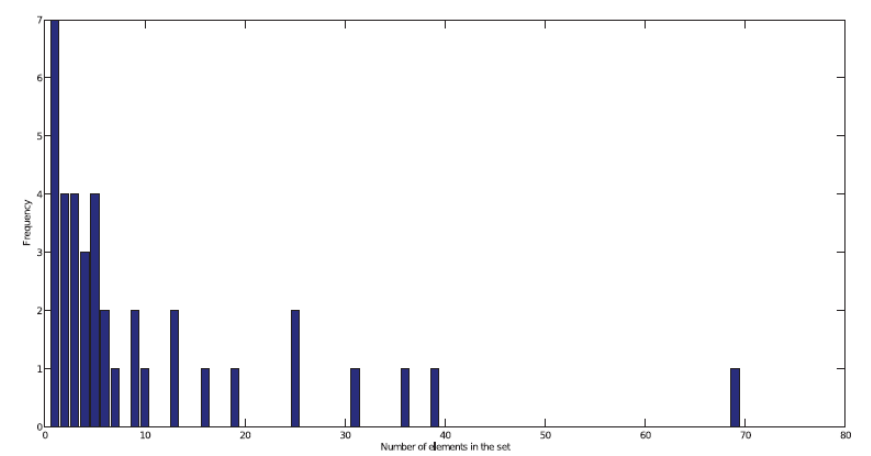

(a) Before

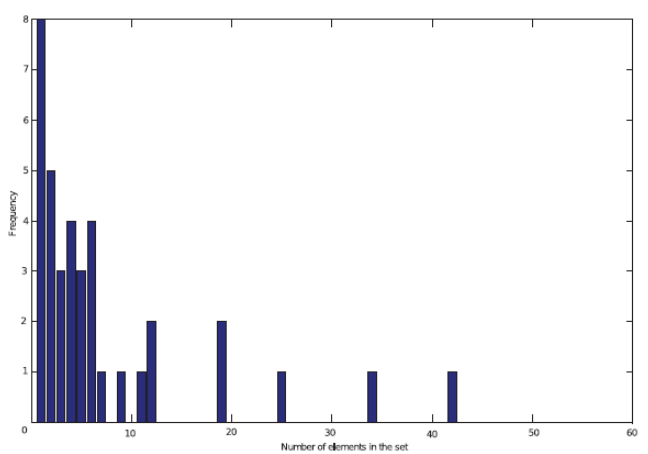

(b) After

Figure 9. Histogram of the Size of the Possible Distances at Receiver before and after filtering with LQI $=80$ (Indoor Experiments).

For example, if the link quality is better, and the LQI is increased to 100, the histogram would be updated as seen in Fig. 10. If some uncertainty about an exact distance still exists, heuristics can then be applied to further decrease the estimated distance sets. Regardless, the reader may clearly note that with $L Q I=100$, the set size is significantly decreased. 


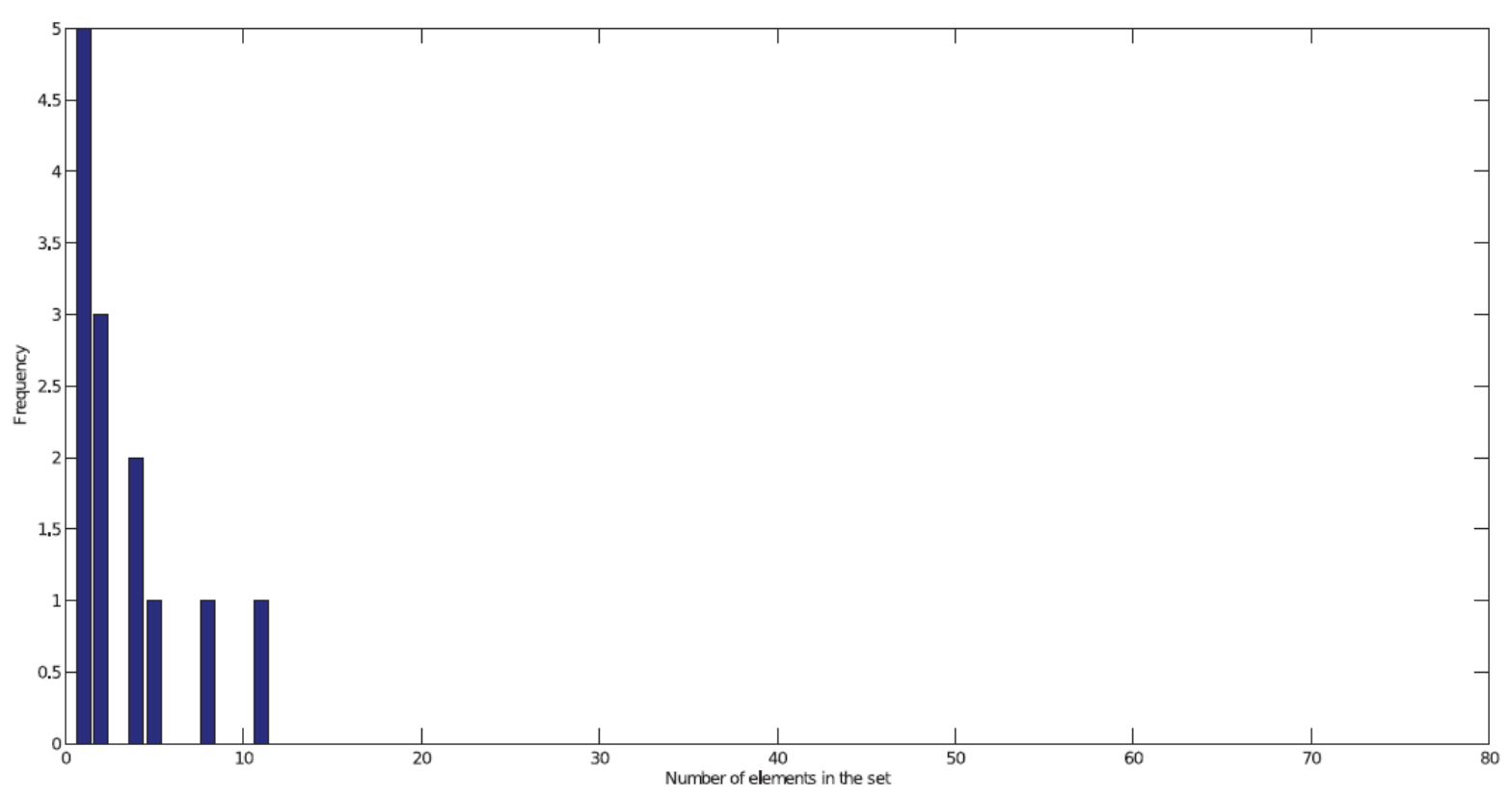

Figure 10. Histogram of the Size of the Possible Distances at Receiver after filtering with LQI $=100$ (Indoor Experiments).

\section{From a simple filtering to a distributed consensus}

So far we have described our distance estimation system that uses the RSSI and LQI metrics to increase the precision in estimating communication distances between any two wireless devices. To test our hypothesis, we set up experiments using sensor nodes. Results indicate that when the LQI is high, our algorithm is capable of significantly increasing the probability of correctly estimating this distance. We then build upon this simple filtering mechanism that does not consider the announced power level of the transmitting device, by adding a "communication layer" to the distance estimation process. That is, nodes will exchange their estimates and set of inferred distances with each other, through a distributed protocol, and merge the associated beliefs as to which distance is correct.

\subsection{Classification of Algorithms}

Distance estimation algorithms are often classified according to whether or not they require specific hardware. Those that do, are often denominated as "range-based", while those that do not, are known as "range-free" [29, 30]. Range-free approaches are generally based on the connectivity information to estimate the location and the distances. The fundamental assumption behind these algorithms is that if two devices are able to communicate, then the distance between them (with high probability) is less than their maximum transmission distance. The distance is estimated through the number of hops between nodes, or based on an Euclidean value [30]. Albeit they are simple and of low cost, range-free approaches are highly dependent on the node density, network topology and well-defined references of devices [31]. 
On the other hand, range-based approaches expect additional hardware or specific circuitry to exist. For example, the manner in which the distance information is obtained, can rely on the time of arrival (ToA), the time-difference of arrival (TDoA), the angle of arrival (AoA), or RSSI and LQI metrics. Both ToA as well as TDoA techniques measure distance between devices using the propagation time of the communication signal. AoA techniques, in addition to using the propagation time, use the antenna's amplitude or phase response to identify the direction of the signal. In this work, we focus on a range-based approach that relies solely on the RSSI and LQI values.

While approaches based on AoA, ToA or TDoA typically achieve higher accuracy than those using the RSSI measurements, in Section 2 we showed that improvements can be made through a complementary combination of RSSI and LQI. In fact, while RSSI can be an attractive agile link estimator, LQI can make more precise estimates with the tradeoff of increased delay and estimation cost [27]. So, as long as appropriate assumptions are made, a combination of LQI and RSSI leads to a useful solution for indoor distance estimation and localization. Additionally, since both RSSI and LQI are commonly present in all commercial off-the-shelf hardware, applicability of such a solution can be universal to almost all hardware network deployment.

\subsection{Characterizing the Experimental Set-Up}

The same experimental setup was used as previously stated in Section 2-A. Additionally, however, other data collecting experiments where run. Thousands of experimental data points were obtained and stored. We called this dataset as the "perfect experimental data" (PED), as it reflects a controlled scenario where obtained RSSI and LQI values are taken with minimal interference, although this dataset is far from "perfect". After the implementation of our algorithm, as well as during the adjustment of it's performance, we used experimentally obtained PED as an input to a MATLAB-based analysis and simulation. The simulation would read results from the PED, and output the estimated distance for each of the simulated device. Random behavior was introduced by arbitrarily choosing the data points within the PED. Incorrect behavior was considered by using input from the PED that originated from different distances. By doing this, we were able to induce an exact amount of failure (percentages) by simply using inputs from the PED that represented the percentage of the distance being simulated.

\section{Our Contribution}

In our proposed indoor distance estimation algorithm, we rely both on the Fuzzy Logic, as well as on the TBM to better deal with the variability and uncertainty of the RSSI and LQI signals.

Details of Algorithm:

\section{First Step - Filtering Distances with RSSI and LQI}

The first step of our proposed algorithm is really just a simple filter to reduce the set size of the possible distances inferred. The idea behind using both the RSSI and the LQI is to increase the correctness of distance estimation and is a direct analysis of the PDE collected. Given varying distances and power levels, we uncovered the fact that, under our experimental scenario, the LQI could be used to filter out possible 
distances. This is true for certain combinations of power vs. distances as the expected best case of LQI could immediately indicate a (possibly) infeasible situation. After calculating the mean of the signals for each transmission power level and distance, we identify an exponential curve to fit the data. So, when a device A decodes the tuple

$$
I_{A}=[\mathrm{RSSI}, \mathrm{LQI}],
$$

the first step of our algorithm returns

$$
\left.D_{A}=\text { [all possible inferred distances given } I_{A}\right] \text {. }
$$

This clearly is a very large set of distances, since we consider all possible transmission powers for the input tuple. For example, in Fig. 11, with the tuple RSSI $=-100 \mathrm{dBm}$ and $\mathrm{LQI}=80$ we obtain the experimental results after executing this first step. It contains the number of sets with unique distances, considering all possible power vs. distances combinations. From this input, our algorithm inferred over 250 different distances. While the original RSSI guess, inferred was over 415.

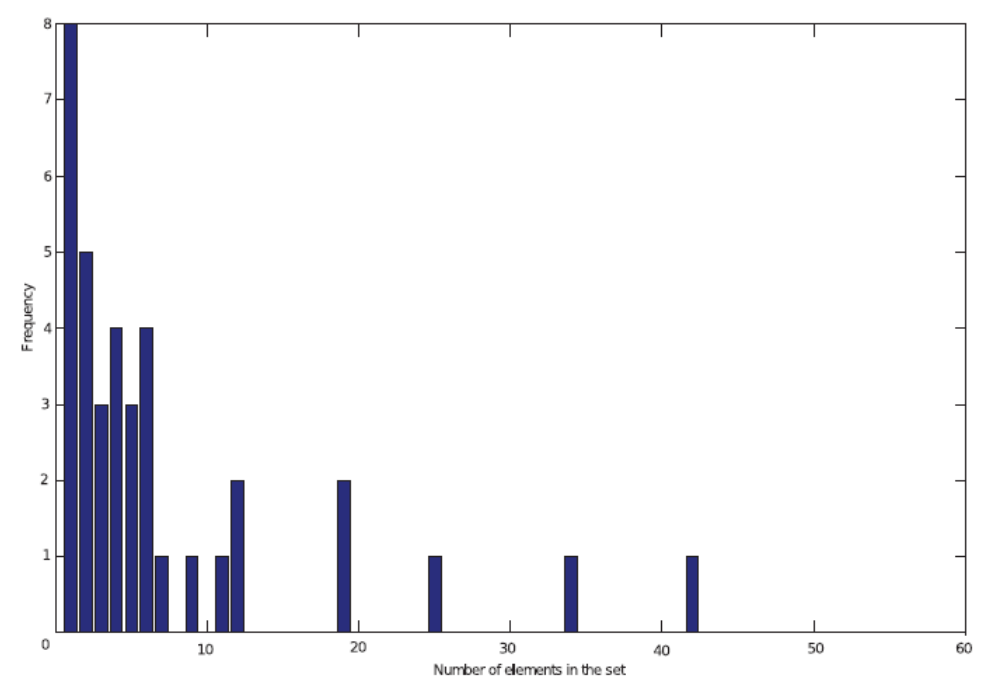

Figure 11. Histogram of the Size of the Possible Distances at Receiver after filtering with LQI $=80$ (Indoor Experiments).

\section{Second Step - Exchanging Sets and Ordering}

The second step of our algorithm consists of ordering the set from the smallest distance to the largest, and having the 2 communicating devices share their sets. That is, devices $\mathrm{A}$ and $\mathrm{B}$, exchange $D_{A}$ and $D_{B}$. After this exchange, both devices will be able to order the set of all distances as

$$
\left.\mathrm{D}=\text { [ordered set including } D_{A} \text { and } D_{B}\right] \text {. }
$$

\section{Third Step - Determining Fuzzy Membership Class Values}

As the third step of our algorithm, we rely on the linguistic idea behind the fuzzy logic, and on the triangular membership functions to identify the number of elements from the ordered set that belong to each of the membership classes. Fuzzy logic is a multi-valued logic that is capable of mapping imprecise terms into crisp values. Impreciseness is represented through the use of linguistic terms. For example, devices may be classified as being "far" from or "close" to one another. Instead of specifying distance in terms of hard thresholds, a linguistic value of "close" become much easier to state and understand. We do not, however, fully utilize the building blocks of fuzzy logic (but refer the interested reader to [25] for further details). 
We divide the set D into approximately 3 smaller overlapping sets, as well as 2 additional sets that represent the overlap among themselves. The first 3 sets represents the linguistic terms "close", "medium" and "far". Fig. 12 may help in this explanation. Let's assume that $d$ contain values that range from 0 to 30 . Approximately the first third of the distances becomes a part of the "close" triangular membership function. The next third becomes "medium", and the last third "far".

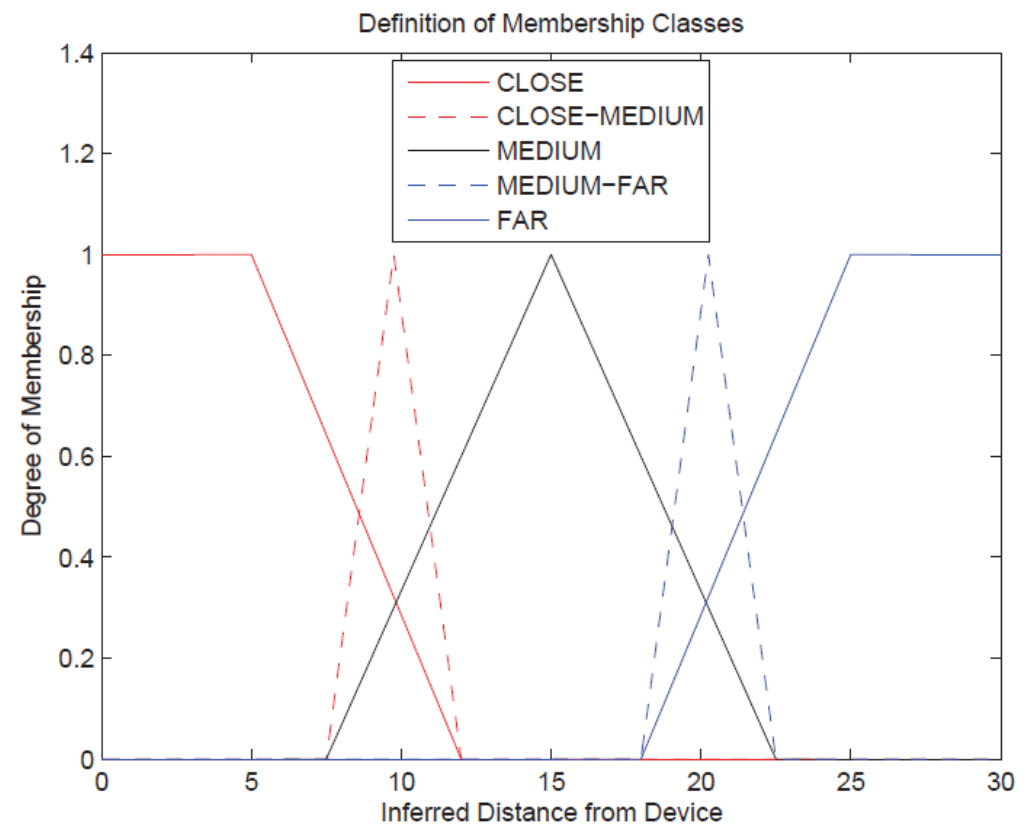

Figure 12. Example of Membership Classes for a Distance Set.

However, the intersections will also have linguistic terms. The one between "close" and "medium" will be denominated "close-medium". While the other will be denominated "medium-far". These 5 membership functions form our fuzzy membership classes.

\section{Fourth Step - Calculating Beliefs using TBM}

With the membership classes defined, the next step is to try to calculate the belief the device has with regards to the classes. We rely on the Transferable Belief Model (TBM) [26] as the tool for this step since it can easily handle uncertainty. In a typical probability theory, a random experiment is one in which the outcome varies in an unpredictable fashion whenever repeated under the same conditions. From each experiment, a set of one or more measurements or observations are made. The formalism introduced by the theory is supposed to model the actual situation. But, the question is what happens when the universe is unknown, or if the user is ignorant about a statement. Does this ignorance necessarily translate into the opposite statement, as assumed in the Bayesian reasoning? A paradigm shift took place in 1976, when Shafer's classical book [32] questioned such arguments. For example, according to Dempster-Schafer's (DS) formalism, if the device believes in something with only $30 \%$ certainty, it does not necessarily mean that it is $70 \%$ certain that it does not believe in it. TBM is a variant of DS that allows one to model and combine evidence in order to make a better decision. It generalizes the Bayesian approach without the need of previously known probabilities. Therefore, it is able to deal with redundant and conflicting information, which is perfect for our need. It is based on the ideas of building beliefs on a subjective knowledge, and by incorporating the information using DS's rule of combination. 
Each device individually counts the number of elements that are within each of the 5 classes. This result in a ratio determined by the belief of that class. For example, assuming there are 40 inferred distances in the distance set $D_{A}$. If, after running the fuzzy logic membership that could identify the number of elements belonging to the "close" class, we arrive at the ratio 8/40. At the end of this step, we have individual beliefs that each device has with regards to each of the 5 membership classes.

\section{Fifth Step - Combining Beliefs using TBM}

Once we calculate the individual device's belief, we merge their beliefs using TBM. TBM assumes a frame of discernment $(\mathrm{FoD}) \Theta$ which contains all possible classes (including exclusive and exhaustive solutions). In our scenario, this represents:

$$
\Theta=[(\text { close }),(\text { medium }),(\text { far })] \text {. }
$$

The power set $2^{\Theta}$ contains all the singleton hypotheses, as well as all the disjunctions. Therefore, it is specified by:

$$
\begin{aligned}
\Theta= & {[(\text { close }),(\text { close }- \text { medium }),(\text { close }- \text { far }),} \\
& (\text { medium }),(\text { medium }- \text { far }),(\text { far }), \\
& (\text { close }- \text { medium }- \text { far })] .
\end{aligned}
$$

A basic belief assignment (bba) is given by $\mathrm{m}: 2 \Theta \rightarrow[0,1]$ with $\Sigma_{\mathrm{A} \subset \Theta} \mathrm{m}(\mathrm{A})=1$, where $\mathrm{m}(\mathrm{A})$ is the basic belief mass (bbm) given to A. Every $A \subset \Theta$ such that $\mathrm{m}(\mathrm{A})>0$ is a focal representation. Every subset that has some (even subjective) support for the real state provides a bbm. This is one of the key points of TBM, such that it can allocate masses to a subset of $\Theta$, rather than depending only on mutually exclusive hypotheses [33].

Beliefs from two devices can be fused or combined using various rules of combination. DS has a normalized rule where the mass assigned to the empty set $\varnothing$ is always zero. The combination process relies on the DS operators. Given two distinct bba's $\mathrm{m}_{\mathrm{A}}$ and $\mathrm{m}_{\mathrm{B}}$ obtained from devices $\mathrm{A}$ and $\mathrm{B}$, combination of their beliefs defined on the same FoD is:

$$
m_{A B}(C)=\Sigma_{(X \cap Y=C)} m_{A}(X) * m_{B}(Y) .
$$

This is a formalism that can model and manage doubts independent of the hypothesis. This is another advantage of this theory as compared to the Bayesian probability. For the reader who is not familiar with the DS and the TBM, we provide a step-by-step formulation of the equations and provide an example of its potential use. When combining the beliefs of two devices A and B with regards to the "close" (clo.) membership class:

$$
\begin{aligned}
\mathrm{m}_{\mathrm{AB}}(\text { clo. })= & \mathrm{m}_{\mathrm{A}}(\text { clo. }) * \mathrm{~m}_{\mathrm{B}}(\text { clo. })+ \\
& \mathrm{m}_{\mathrm{A}}(\text { close }- \text { medium }) * \mathrm{~m}_{\mathrm{B}}(\text { clo. })+ \\
& \mathrm{m}_{\mathrm{A}}(\text { clo }) * \mathrm{~m}_{\mathrm{B}}(\text { close }- \text { medium }) .
\end{aligned}
$$

When combining the beliefs of two devices A and B with regards to the "medium" (med.) membership class:

$$
\begin{aligned}
\mathrm{m}_{\mathrm{AB}}(\text { med. })= & \mathrm{m}_{\mathrm{A}}(\text { med. }) * \mathrm{~m}_{\mathrm{B}}(\text { med. })+ \\
& \mathrm{m}_{\mathrm{A}}(\text { close }- \text { medium }) * \mathrm{~m}_{\mathrm{B}}(\text { med. })+ \\
& \mathrm{m}_{\mathrm{A}}(\text { medium }- \text { far }) * \mathrm{~m}_{\mathrm{B}}(\text { med } .)+
\end{aligned}
$$




$$
\begin{aligned}
& \mathrm{m}_{\mathrm{A}}(\text { med. }) * \mathrm{~m}_{\mathrm{B}}(\text { close }- \text { medium })+ \\
& \mathrm{m}_{\mathrm{A}}(\text { medium }- \text { far }) * \\
& \mathrm{~m}_{\mathrm{B}}(\text { close }- \text { medium })+ \\
& \mathrm{m}_{\mathrm{A}}(\text { close }- \text { medium }) * \\
& \mathrm{~m}_{\mathrm{B}}(\text { medium }- \text { far })+ \\
& \mathrm{m}_{\mathrm{A}}(\text { med. }) * \mathrm{~m}_{\mathrm{B}}(\text { medium }- \text { far })
\end{aligned}
$$

When the beliefs of two devices A and B are combined with regards to the "far" membership class:

$$
\begin{aligned}
\mathrm{m}_{\mathrm{AB}}(\mathrm{f} \text { ar })= & \mathrm{m}_{\mathrm{A}}(\text { far }) * \mathrm{~m}_{\mathrm{B}}(\text { far })+ \\
& \mathrm{m}_{\mathrm{A}}(\text { medium }- \text { far }) * \mathrm{~m}_{\mathrm{B}}(\text { far })+ \\
& \mathrm{m}_{\mathrm{A}}(\text { far }) * \mathrm{~m}_{\mathrm{B}}(\text { medium }- \text { far }) . .
\end{aligned}
$$

By calculating the TBM combination of all the membership classes ("close", "medium" and "far"), the output of this fifth step would be the class with highest membership value. Notably, having this naming convention based on the linguistic terms helps us readily perceive how far away the nodes are from each other.

\section{Validation based on experimental values}

In this Section, we show the distance estimation results between devices that rely on our proposed algorithm. It may be noted that the results are based on real experimental data (i.e., PED). We describe both the failure-free results (where data used is from the exact distance where it was collected), as well as failure-induced results (when the data are from different collection distances). For a given RSSI and LQI, we run our algorithm and obtain the resulting estimated distance. We then compare if that distance is within certain linguistic bounds from the known distance. We calculate the success rate by calculating the number of times that our algorithm detects correctly if the nodes are "close", "medium" or "far" away from each other.

The linguistic terms are well defined - in that they represent distances while the exact value ranges of each linguistic term depends on the unique elements of the exchanged $\mathrm{D}_{\mathrm{A}}$ and $\mathrm{D}_{\mathrm{B}}$ distances. In this aspect, the ranges within the membership classes of "close", "medium" or "far" could dynamically vary. This is very important to emphasize. For this reason, we have also plotted the mean of the range difference between the actual and the inferred values when our algorithm correctly judges the distances, as well as the range when the algorithm incorrectly infers the distances. This is represented through the percentages of actual distance. As we describe individual experimental results, these graphs can be better understood.

With regards to the simulation environment, as previously stated, the input used comes from the PED files with the signals obtained from the experimental measurements. The MATLAB [34] code reads the input files and calculates both the fuzzy-membership functions, as well as the TBM merging and analysis of uncertainty. More details can be seen in Table I. 


\section{Macrothink}

Table 1. Simulation Parameters.

\begin{tabular}{|l|c|}
\hline \multicolumn{1}{|c|}{ Parameter } & Values \\
\hline \hline Distance & $0 \mathrm{~cm}-100 \mathrm{~cm}$ \\
\hline Number of Simulation Runs & 100 for every instance \\
\hline Failure Probability & $0 \%-90 \%$ \\
\hline
\end{tabular}

\subsection{Failure-Free Experimental Results}

Our first set of experimental analysis considered using the experimental PED data from the exact location where it is measured. This means if the experiment measured the RSSI and LQI signals at $300 \mathrm{~cm}$, then for all the simulations between two communicating devices, we would randomly read from the $300 \mathrm{~cm}$ file previously stored as the measured signals. We call this a "failure-free" scenario because the input values used are (supposedly) "perfect". However, given the known variations in RSSI and LQI when measured indoors, this is far from true. In fact, the correctness of our algorithm given various distances can be seen in Fig. 13.

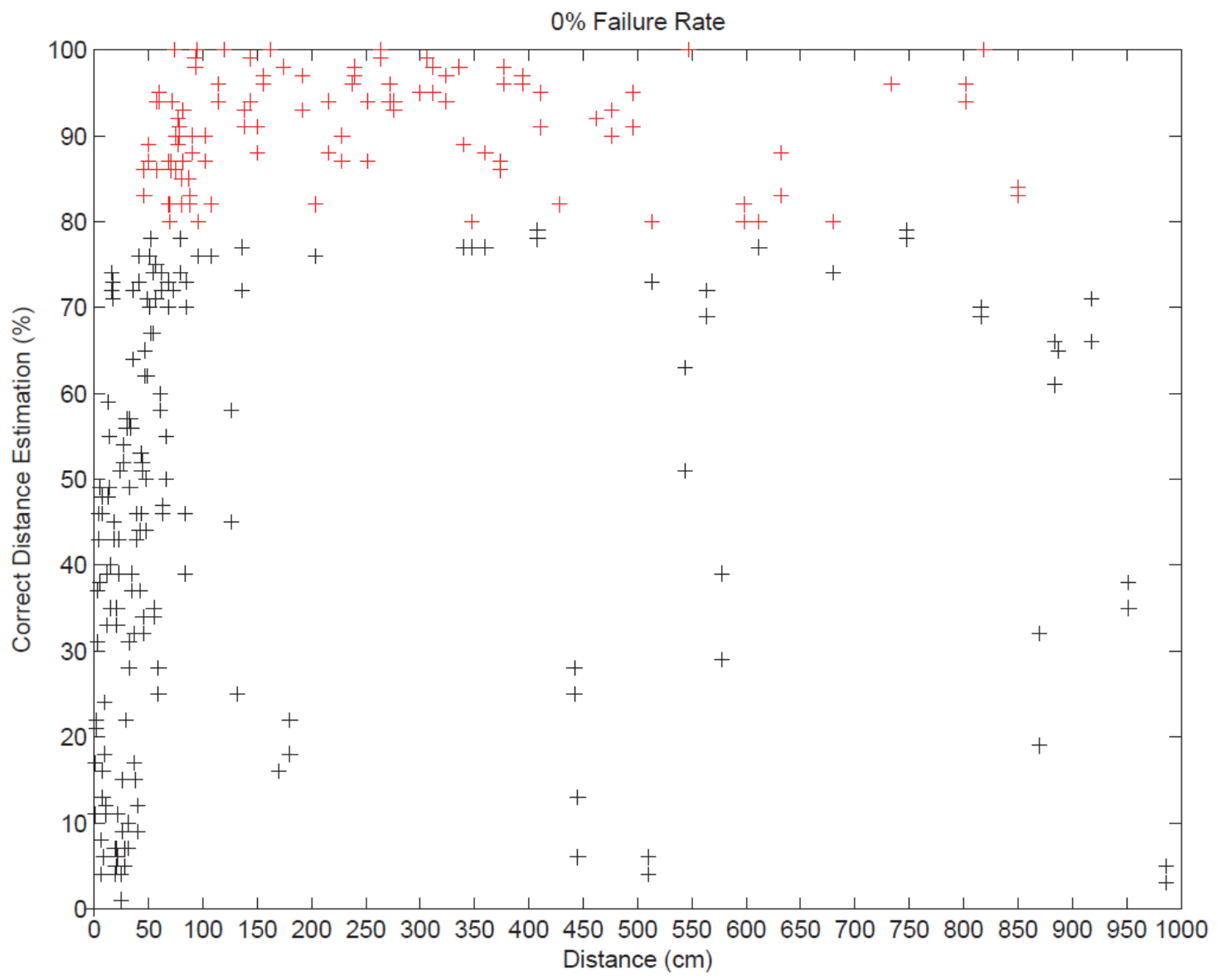

Figure 13. Results of Distance Estimation when in Failure-Free Scenario.

The $\mathrm{x}$-axis of our graphs identifies the actual experimental distance where the signals are measured. Although our experiments included distances over $25 \mathrm{~m}$, in this paper, we have 


\section{Al Macrothink}

limited the data points from 0 to 10 meters. The y-axis on Fig. 13 shows the correct distance estimation of the failure-free runs. We have highlighted data points whenever the correct distance estimation is above $80 \%$ (in red). In our opinion, this percentage is one that most system designers might be comfortable with in a real deployment. Incorrect distances are inferred at both very short distances $(<50 \mathrm{~cm})$ as well as when they are further away. Although, given the random aspect of the simulations, there are moments when devices are considerably further apart ( $>7 \mathrm{~m})$ where distances are correctly inferred over $97 \%$ of the time.

To better understand how well our algorithm works, we simultaneously calculated the average error between the actual distance and the inferred distance. We denominated this as a "spread", and Fig. 14(a) and 14(b) respectively illustrate an exact average as well as the relative average (with regards to the current distance). While for most of the cases the spread maintains itself around the $15 \mathrm{~cm}$ mark, this obviously has a higher impact on the error and inaccuracy when the distances are smaller. However, as can be seen in Fig. 14(b), this error slowly tapers off from as high as $100 \%$, to very low percentages $(<5 \%)$.

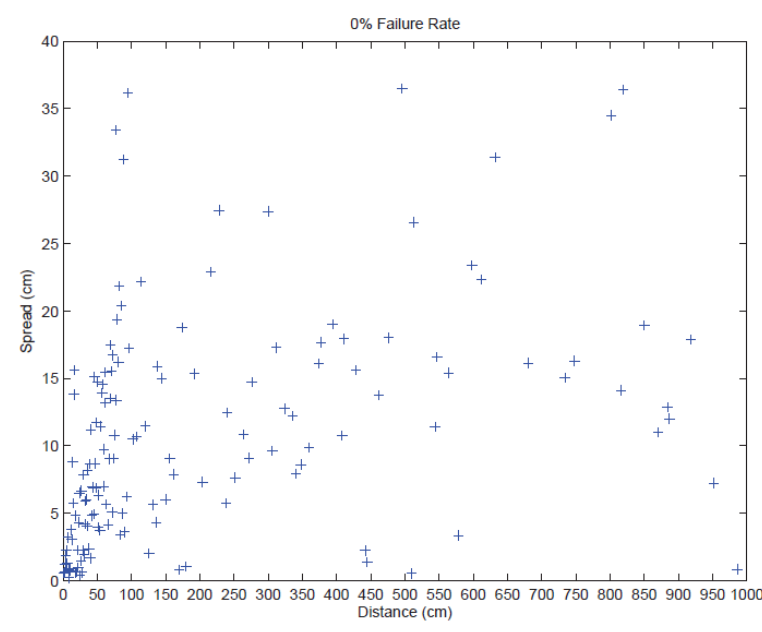

(a) In $\mathrm{cm}$

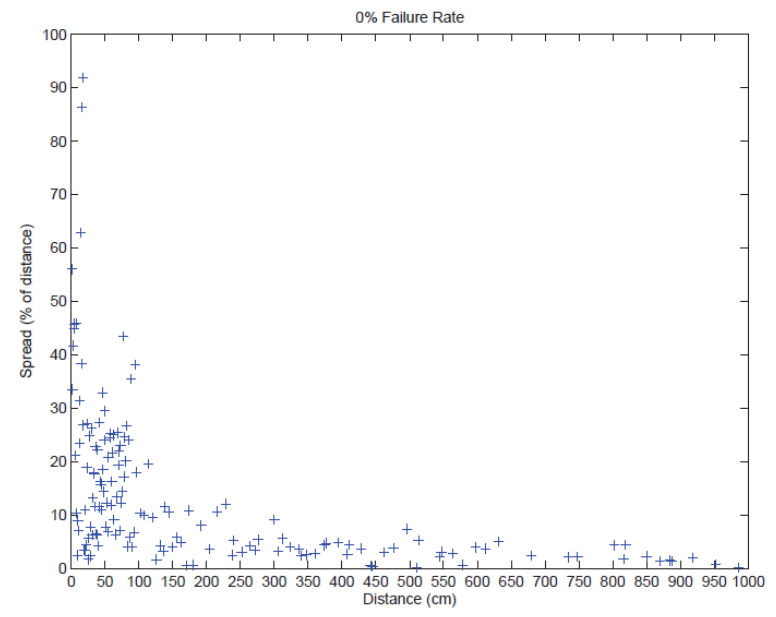

(b) In percentage of actual distance

Figure 14. Average Spread of Inferred Distances.

In a similar manner, we have also plotted the results of the spread when our algorithms incorrectly infers the distance. In this case, what Fig. 15(a) and 15(b) show is how bad the errors are whenever they occur. 


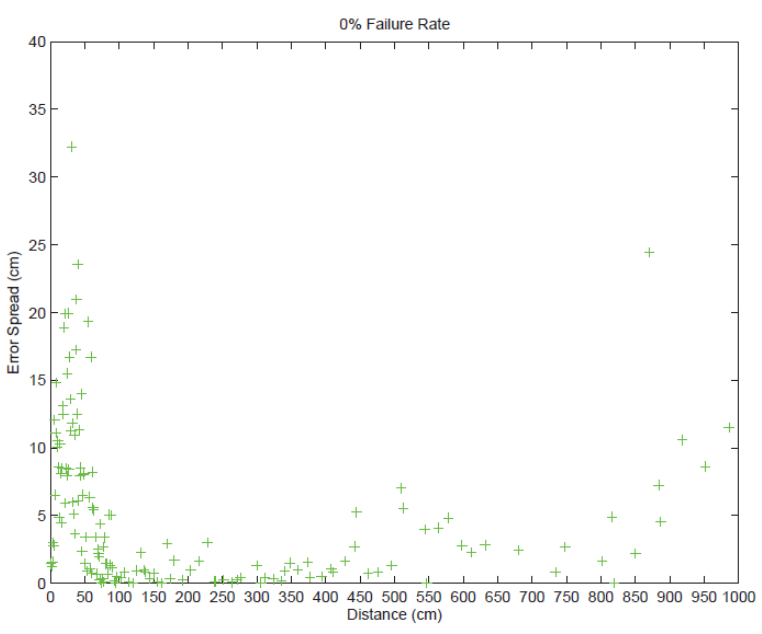

(a) In $\mathrm{cm}$

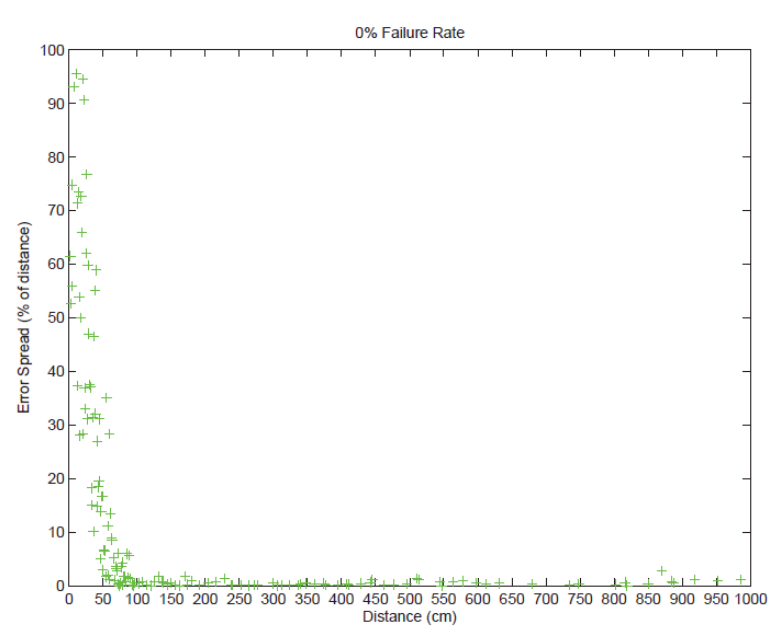

(b) In percentage of actual distance

Figure 15. Average Error Spread of Inferred Distances.

It may be noted that while our algorithm does not deal well with very short distances $(<$ $50 \mathrm{~cm}$ ), results are actually very good above that range. So, considering the fact that we used experimental data in an environment and scenario with minimal interference and wild fluctuations in the RSSI and LQI signals, we thought that a better way is to observe the performance of our algorithm when failures are present.

\subsection{Failure-Induced Experimental Results}

In this Section, we describe the behavior of our algorithm as failures are added. As we introduce failures, it is expected that the performance decreases, and more to our algorithm infers the incorrect distance. This can be clearly noted in Fig. 16. Although we later provide (in Fig. 19) all the plots from 10\% - 90\% induced failures, we would like to focus on 3 cases: $20 \%, 50 \%$ and $90 \%$ of failures. 


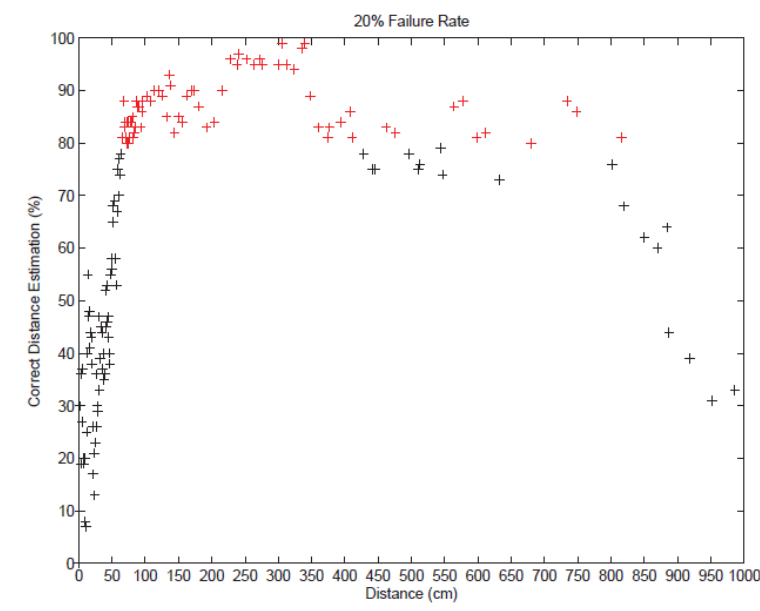

(a) $20 \%$ Failure

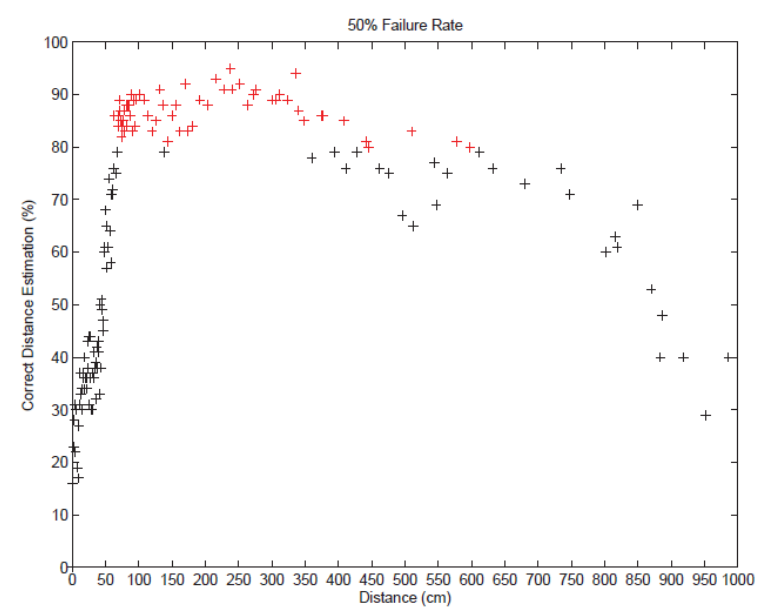

(b) $50 \%$ Failure

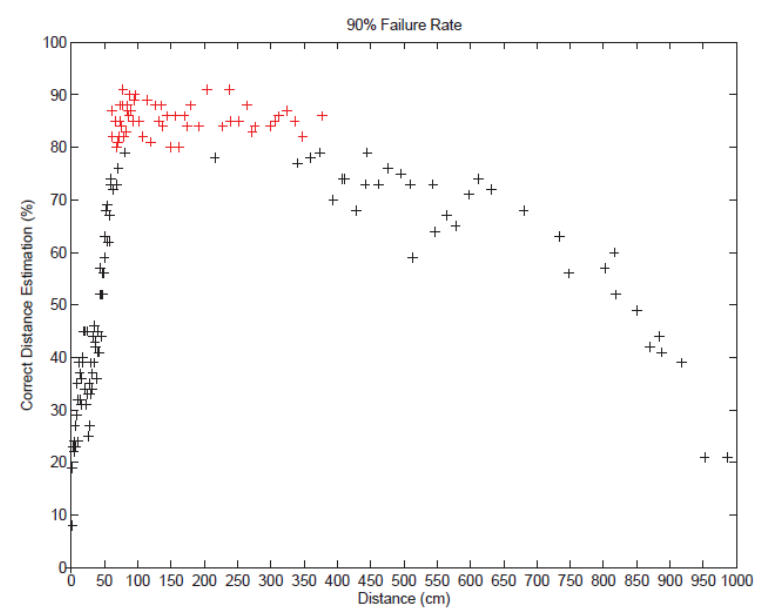

(c) $90 \%$ Failure

Figure 16. Correct Distance Estimation as Failures are Introduced.

From Fig. 16, we notice a reduction in the number of instances of correct detections above the $80 \%$ threshold, but, within the $100 \mathrm{~cm}$ to approximately $400 \mathrm{~cm}$ even with high failure rates. The combination of fuzzy logic and TBM is able to consider the uncertain beliefs of each device before deciding on the distance between them. These results clearly show that the combination of RSSI and LQI used in our algorithms can be successfully used in a practical scenario.

Due to the close similarities between most of the graphs among all failure percentages, we will show the spread of only the $20 \%$ and $90 \%$ cases in Fig. 17. As can be seen, the spread with the $100-400 \mathrm{~cm}$ range is consistently found between $15 \%-5 \%$ of the actual distance, respectively. 


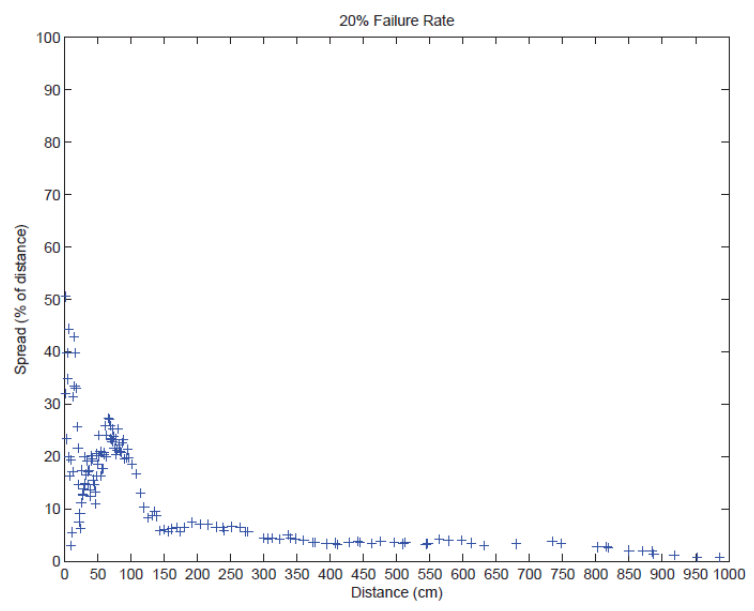

(a) $20 \%$ Failure

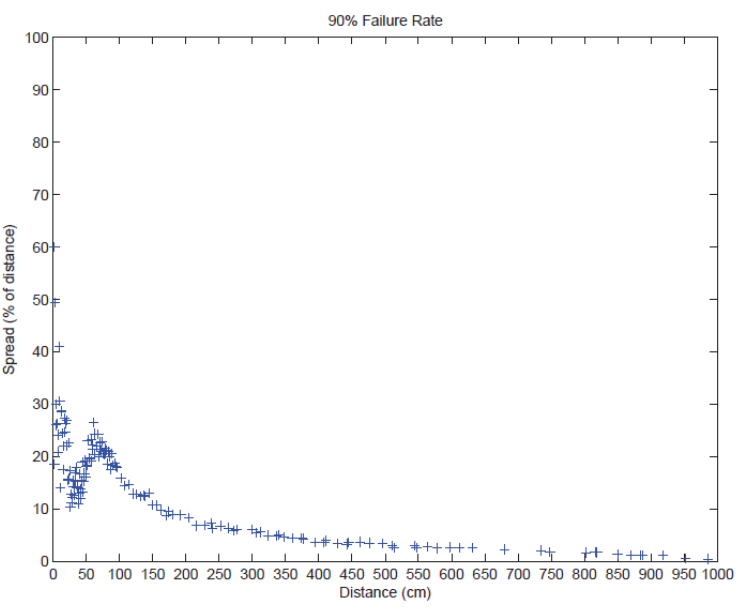

(b) $90 \%$ Failure

Figure 17. Average Spread as Failures are Introduced.

Similarly, we show the results of the error spread in Fig. 18. As expected, the errors are greater when we induce $90 \%$ failures. Otherwise, the overall behavior is very consistent.

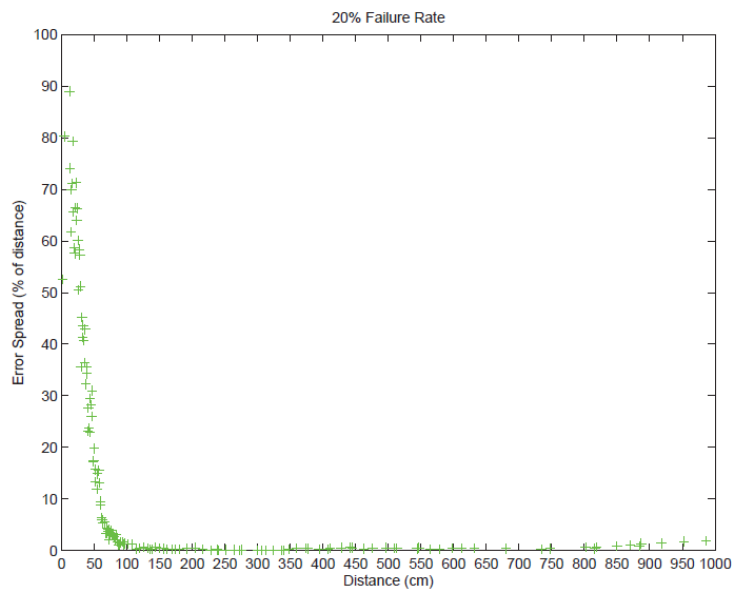

(a) $20 \%$ Failure

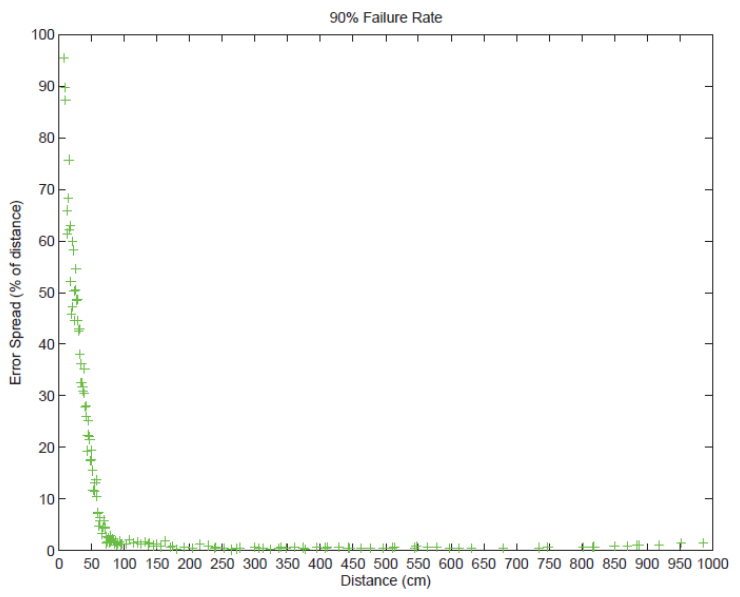

(b) $90 \%$ Failure

Figure 18. Average Error Spread as Failures are Introduced. 


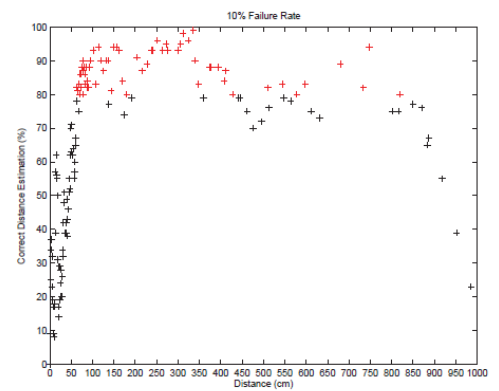

(a) $10 \%$ Failure

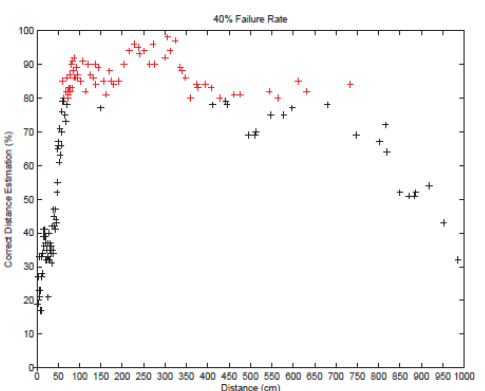

(d) $40 \%$ Failure

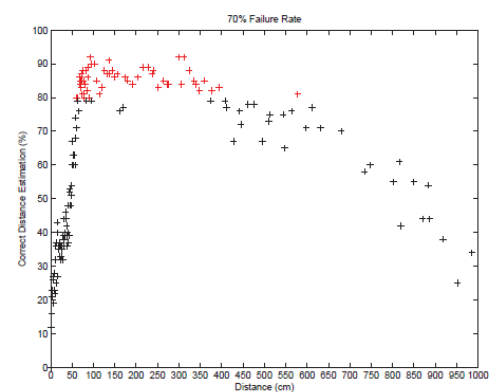

(g) $70 \%$ Failure

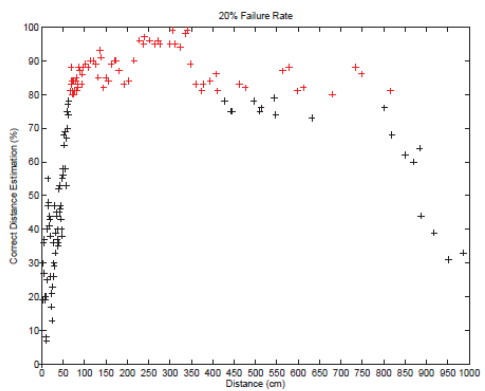

(b) $20 \%$ Failure

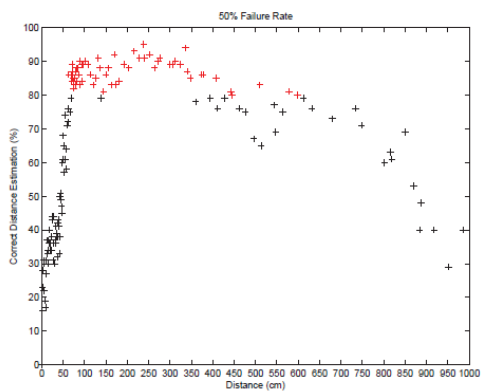

(e) $50 \%$ Failure

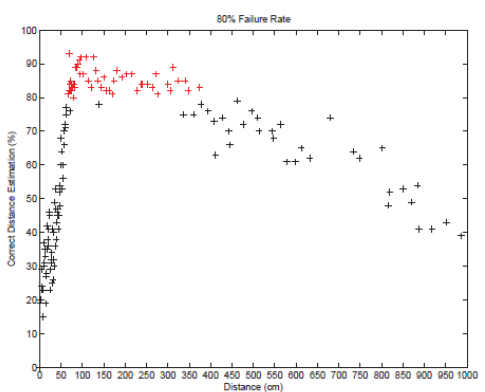

(h) $80 \%$ Failure

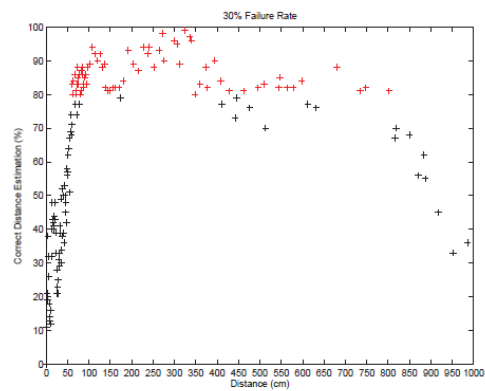

(c) $30 \%$ Failure

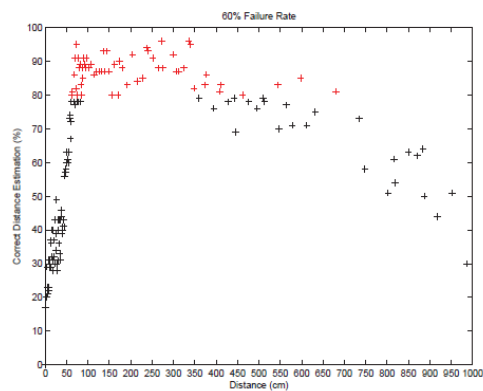

(f) $60 \%$ Failure

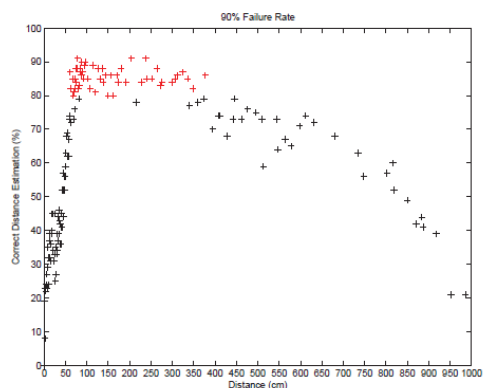

(i) $90 \%$ Failure

Figure 19. Correct Distance Estimation as Failures are Introduced. 


\section{Conclusion}

Although the received signal strength indicator (RSSI) and on the link quality indicator (LQI) have previously been individually shown to be largely inadequate as a distance estimator for indoor scenarios, this paper proposes an algorithm that relies exactly on these two parameters to correctly infer distances between devices. While not denying previous experimental research which have correctly shown that they behave inconsistently, even in an ideal scenario - what we have done is embraced the inherent uncertainty of these signals. Initially, we developed a simple filtering mechanism that does not consider the announced power level of the transmitting device, but rather the RSSI and LQI values. This possibly large set of inferred distances are then exchanged, and run through a distributed protocol to merge the associated beliefs.

The process of selecting the distance with the maximum likelihood of their occurrence goes through a set of fuzzy logic rules before being combined with the help of the transferable belief model (TBM) which enables us to model and combine evidence in order to make an improved decision. This unique combination is able to deal with redundant and conflicting information since it is based on the ideas of building beliefs on a subjective knowledge. Experimental analysis of this novel approach is observed to correctly infer distances between devices in the range of $100 \mathrm{~cm}$ to $400 \mathrm{~cm}$ in an indoor scenario, for at least $80 \%$ of the times and reaching accuracy as high as $100 \%$.

\section{References}

[1] H. Hashemi, "The indoor radio propagation channel," in Proceedings of the IEEE, vol. 81, July 1993.

[2] J. Zhao and R. Govindan, "Understanding packet delivery performance in dense wireless sensor networks," in Proceedings of the $1^{\text {st }}$ international conference on Embedded networked sensor systems, SenSys '03, Los Angeles, CA, USA, November 5-7, 2003.

[3] S. Hussain and M. S. Rahman, "Using received signal strength indicator to detect node replacement and replication attacks in wireless sensor networks," in Proc. SPIE, no. 7344, 73440G (April 13, 2009). http://dx.doi.org/10.1117/12.824207

[4] K. Chintalapudi, A. P. Iyer, and V. N. Padmanabhan, "Indoor localization without the pain," in Proceedings of the sixteenth annual international conference on Mobile computing and networking, MobiCom '10, Chicago, IL, USA, September 20 - 24, 2010 pp. 173-184. http://dx.doi.org/10.1145/1859995.1860016

[5] H. Miura, K. Hirano, N. Matsuda, H. Taki, N. Abe, and S. Hori, "Indoor localization for mobile node based on rssi," in Knowledge-Based Intelligent Information and Engineering Systems (B. Apolloni, R. Howlett, and L. Jain, eds.), vol. 4694 of Lecture Notes in Computer Science, pp. 1065-1072, 2007.

[6] G. Zanca, F. Zorzi, A. Zanella, and M. Zorzi, "Experimental comparison of rssi-based localization algorithms for indoor wireless sensor networks," in Proceedings of the workshop on Real-world wireless sensor networks, REALWSN '08, Glasgow, Scotland Uk, April 01 - 04, 2008. http://dx.doi.org/10.1145/1435473.1435475 
[7] K. Whitehouse, C. Karlof, A. Woo, F. Jiang, and D. E. Culler, "The effects of ranging noise on multihop localization: an empirical study," in Proceedings of the 4th international symposium on Information processing in sensor networks (IPSN '05), pp. 73-80, UCLA, Los Angeles, California, USA. April 25-27, 2005.

[8] A. Savvides, H. Park, and M. B. Srivastava, "The bits and flops of the n-hop multilateration primitive for node localization problems," in Proceedings of the First ACM International Workshop on Wireless Sensor Networks and Applications, (WSNA 2002), Atlanta, GA, USA, September 28, 2002. Pp. 112-121.

[9] B. Li and H. Wang, "A low complexity localization algorithm in wireless sensor network," in Proceedings of the 2010 International Conference on Innovative Computing and Communication and 2010 Asia-Pacific Conference on Information Technology and Ocean Engineering, CICC-ITOE '10, Macao, 30-31 Jan. 2010. Pp. 217-220, http://dx.doi.org/10.1109/CICC-ITOE.2010.62

[10]F. Potorti, A. Corucci, P. Nepa, F. Furfari, P. Barsocchi, and A. Buffi, "Accuracy limits of in-room localisation using RSSI," in IEEE International Symposium of Antennas and Propagation Society (APSURSI '09), Charleston, SC, USA. 1-5 June 2009. pp. 1-4, http://dx.doi.org/10.1109/APS.2009.5172087

[11]X. X. Ji Luo and Q. Zhang, "Understanding link feature of wireless sensor networks in outdoor space: A measurement study," in Proceedings of the IEEE Global Communications Conference (GLOBECOM 2011), 5-9 December 2011, Houston, Texas, USA, pp. 1-5. http://dx.doi.org/10.1109/GLOCOM.2011.6134117

[12] A. S. Paul and E. A. Wan, "Rssi-based indoor localization and tracking using sigma-point kalman smoothers," IEEE Journal of Selected Topics in Signal Processing, vol. 3, pp. 860-873, October 2009. http://dx.doi.org/10.1109/JSTSP.2009.2032309

[13] M. M. Noel, P. P. Joshi, and T. C. Jannett, "Improved maximum likelihood estimation of target position in wireless sensor networks using particle swarm optimization," in Proceedings of the Third International Conference on Information Technology: New Generations, (ITNG 2006), 10-12 April 2006, Las Vegas, Nevada, USA. pp. 274-279.

[14]R. Peng and M. L. Sichitiu, "Probabilistic localization for outdoor wireless sensor networks," SIGMOBILE Mob. Comput. Commun. Rev., vol. 11, pp. 53-64, January 2007.

[15]E. Elnahrawy, X. Li, and R. P. Martin, "The limits of localization using RSS," in Proceedings of the 2nd International Conference on Embedded Networked Sensor Systems (SenSys 2004), Baltimore, MD, USA, November 3-5, 2004, pp. 283-284.

[16] A. T. Parameswaran, M. I. Husain, and S. Upadhyaya, "Is rssi a reliable parameter in sensor localization algorithms an experimental study," in Proceedings of the Field Failure Data Analysis Workshop (F2DA), part of the 28th IEEE Symposium on Reliable Distributed Systems (SRDS 2009), September 27-30, 2009. Niagara Falls, New York, U.S.A., pp. 1-5.

[17]K. Srinivasan and P. Levis, "RSSI is under appreciated," in Proc. 3rd Workshop on Embedded Networked Sensors (EmNets 2006), Cambridge, MA, May 2006.

[18]C. Renner, S. Ernst, C. Weyer, and V. Turau, "Prediction accuracy of link-quality estimators," in Proceedings of the 8th European conference on Wireless sensor networks, 
EWSN'11, Bonn, Germany, on February 23-25, 2011.

[19] N. Baccour, A. Koubaa, H. Youssef, M. B. Jamøa, D. do Rosrio, M. Alves, and L. B. Becker, "F-lqe: A fuzzy link quality estimator for wireless sensor networks," in EWSN'10, Coimbra, Portugal, February 17-19, 2010. pp. 240-255.

[20]X. Kuang and H. Shao, "Maximum likelihood localization algorithm using wireless sensor networks," in Proceedings of the First International Conference on Innovative Computing, Information and Control - Volume 3, (ICICIC '06), Beijing (China). Aug. 30 - Sept. 1 2006. Pp. 263-266. http://dx.doi.org/10.1109/ICICIC.2006.470

[21] M. Mendalka, L. Kulas, and K. Nyka, "Localization in wireless sensor networks based on zigbee platform," in Proceedings of the $17^{\text {th }}$ International Conference on Microwaves, Radar and Wireless Communications (MIKON 2008), Wroclaw (Poland), 19-21 May 2008. Pp. 1-4,

[22]W. Da-peng, W. Mu-qing, and Z. Yan, "Reliable routing mechanism based on neighbor stability for manet," Journal of China Universities of Posts and Telecom, vol. 16, 2009.

[23] S.-Y. Chiang and J.-L. Wang, "Localization in wireless sensor networks by fuzzy logic system," in Proceedings of the 13th International Conference on Knowledge-Based and Intelligent Information and Engineering Systems: Part II, KES '09, Santiago, Chile, September 28-30, 2009, Proceedings, Part II. Lecture Notes in Computer Science Vol. 5712, 2009, pp. 721-728.

[24]X. Feng, Z. Gao, M. Yang, and S. Xiong, "Fuzzy distance measuring based on rssi in wireless sensor network," in Proceedings of the 3rd International Conference on Intelligent System and Knowledge Engineering, (ISKE 2008), Xiamen, China, , 17-19 Nov. 2008. Pp. 395-400.

[25]L. A. Zadeh, "Fuzzy logic and approximate reasoning," Synthese, vol. 30, no. 3-4, pp. 407-428, April/May 1975. http://dx.doi.org/10.1007/BF00485052

[26]P. Smets and R. Kennes, "The transferable belief model," Artificial Intelligence, vol. 66, no. 2, pp. 191-234, 1994. http://dx.doi.org/10.1016/0004-3702(94)90026-4

[27]K. Srinivasan, Towards a wireless lexicon. Dissertation, Electrical Engineering, Stanford University, 2010

[28] Y. Chen and A. Terzis, "On the mechanisms and effects of calibrating rssi measurements for 802.15.4 radios," in 7th European Conference EWSN 2010, Coimbra, Portugal, February 17-19, 2010. Pp. 256-271. http://dx.doi.org/10.1007/978-3-642-11917-0_17

[29] Y. ling Hsieh and K. Wang, "Efficient localization in mobile wireless sensor networks," in Proc. of IEEE International Conference on Sensor Networks Ubiquitous and Trustworthy Computing, vol. 1, Taichung, China, 5-7 June 2006. Pp. 292-297

[30] L. M. Brito and L. M. Peralta, "Collaborative localization in wireless sensor networks," in Proceedings of the 2007 International Conference on Sensor Technologies and Applications, SENSORCOMM '07, Valencia, Spain. 14-20 October 2007, pp. 94-100. http://dx.doi.org/10.1109/SENSORCOMM.2007.36

[31]G. Mao, B. Fidan, and B. D. O. Anderson, "Wireless sensor network localization techniques," Computer Networks, vol. 51, pp. 2529-2553, July 2007. http://dx.doi.org/10.1016/j.comnet.2006.11.018

[32] G. Shafer, A mathematical theory of evidence. Princeton university press, 1976. 
[33] G. Powell, D. Marshall, P. Smets, B. Ristic, and S. Maskell, "Joint tracking and classification of airbourne objects using particle filters and the continuous transferable belief model," in Proceedings of the 9th International Conference on Information Fusion, Florence, Italy. 10-13 July 2006. Pp. 1-8, http://dx.doi.org/10.1109/ICIF.2006.301718 [34] MATLAB, version 7.10.0 (R2010a). Natick, Massachusetts: The MathWorks Inc., 2010.

\section{Copyright Disclaimer}

Copyright reserved by the author(s).

This article is an open-access article distributed under the terms and conditions of the Creative Commons Attribution license (http://creativecommons.org/licenses/by/3.0/). 